\title{
MOTION OF ELASTIC THIN FILMS BY ANISOTROPIC SURFACE DIFFUSION WITH CURVATURE REGULARIZATION
}

\author{
I. FONSECA, N. FUSCO, G. LEONI, M. MORINI
}

Keywords: Minimizing movements, epitaxially strained elastic films, surface diffusion

\begin{abstract}
Short time existence, uniqueness, and regularity for a surface diffusion evolution equation with curvature regularization are proved in the context of epitaxially strained twodimensional films. This is achieved by using the $H^{-1}$-gradient flow structure of the evolution law, via De Giorgi's minimizing movements. This seems to be the first short time existence result for a surface diffusion type geometric evolution equation in the presence of elasticity.
\end{abstract}

\section{INTRODUCTION}

In this paper we study the morphologic evolution of anisotropic epitaxially strained films, driven by stress and surface mass transport. This can be viewed as the evolutionary counterpart of the static theory developed in $[10,19,21,18]$.

We briefly recall the physical mechanism behind the evolution equation. The free interface is allowed to evolve via surface mass transport under the influence of a chemical potential $\mu$. Mass transport in the bulk can be neglected, as it occurs at a much faster time scale (see [30]). According to the Einstein-Nernst relation, the surface flux of atoms is proportional to the tangential gradient of the chemical potential, whose divergence, in turn, equals the rate at which material is removed from or deposited on the interface, due to mass conservation. Thus, we get the volume preserving evolution law

$$
V=C \Delta_{\Gamma} \mu
$$

where $C>0, V$ denotes the normal velocity of the evolving interface $\Gamma, \Delta_{\Gamma}$ stands for the tangential laplacian, and the chemical potential $\mu$ is given by the first variation of the free-energy functional.

In the case of three-dimensional epitaxially strained films with planar symmetries, the underlying model becomes two-dimensional and the free energy functional is given by

$$
\int_{\Omega_{h}} Q(E(u)) d z+\int_{\Gamma_{h}} g(\theta) d \mathcal{H}^{1},
$$

where $h$ is the function whose graph $\Gamma_{h}$ describes the evolving profile of the film, $\Omega_{h}$ is the region occupied by the film, $u$ is displacement of the material, which is assumed to be in (quasistatic) elastic equilibrium at each time, and $\mathcal{H}^{1}$ denotes the one-dimensional measure on $\Gamma_{h}$. Finally, $g$ is an anisotropic surface energy density, evaluated at the angle $\theta$ that the outward surface normal $\nu$ forms with the $x$-axis. The first variation of (1.2) can be written as the sum of three contributions: A constant Lagrange multiplier related to mass conservation, the (anisotropic) curvature of the surface, and the elastic energy density evaluated at the displacement of the solid on the profile of the film. Hence, (1.1) takes the form (assuming $C=1$ )

$$
V=\left(\left(g_{\theta \theta}+g\right) k+Q(E(u))\right)_{\sigma \sigma},
$$

where $k$ is the curvature of $\Gamma_{h},(\cdot)_{\sigma}$ stands for the tangential derivative along $\Gamma_{h}$, and $u(\cdot, t)$ is the elastic equilibrium in $\Omega_{h(\cdot, t)}$, i.e., the minimizer of the elastic energy under the prescribed 
periodicity and boundary conditions (see (1.7) below). The corresponding evolution without surface diffusion, which reduces to

$$
V=\left(g_{\theta \theta}+g\right) k+Q(E(u)),
$$

has been studied by several authors under the assumption that $g_{\theta \theta}+g>0$, i.e., the equation is parabolic (see [25] and the references therein). However, a highly anisotropic non-convex interfacial energy may lead to the existence of certain directions $\theta$ at which the coefficient $g_{\theta \theta}+g$ becomes negative. This is a situation often present in the materials science literature. In this case, equations (1.3) and (1.4) are backward parabolic and the corresponding initial value problem is ill-posed.

To overcome the ill-posedness of the evolution equation, a common approach found in the literature is to regularize higher order terms, i.e, to consider in (1.2) a curvature-dependent surface energy. This was first suggested by Herring ([26]) on physical grounds and later was adopted in [7] and [15], in the particular case of a surface energy of the form

$$
g(\theta, k)=g(\theta)+\frac{\varepsilon}{2} k^{2}
$$

with $\varepsilon$ a positive constant. The evolution without surface diffusion (1.4) is then replaced by

$$
V=\left(g_{\theta \theta}+g\right) k+Q(E(u))-\varepsilon\left(k_{\sigma \sigma}+\frac{1}{2} k^{3}\right),
$$

while in the context of surface diffusion, in view of (1.1), we have the volume preserving evolution law

$$
V=\left(\left(g_{\theta \theta}+g\right) k+Q(E(u))-\varepsilon\left(k_{\sigma \sigma}+\frac{1}{2} k^{3}\right)\right)_{\sigma \sigma} .
$$

This equation was already proposed in [24] for the case without elasticity, and it was studied numerically in [32] for the evolution of voids in elastically stressed materials (see also [31, 11] and references therein). However, to the best of our knowledge no analytical results exist in the literature for equations (1.3), (1.5), and (1.6). Related analytical results concerning the diffuse interface version of such equations may be found, for instance, in [22, 23].

In this paper we prove short time existence, uniqueness, and regularity of a spatially periodic solution to (1.6) in the context of the epitaxially strained two dimensional elastic films over a rigid substrate. Precisely, for $b>0$ we construct a local in time solution of the Cauchy problem

$$
\left\{\begin{array}{l}
\frac{1}{J} \frac{\partial h}{\partial t}=\left(\left(g_{\theta \theta}+g\right) k+Q(E(u))-\varepsilon\left(k_{\sigma \sigma}+\frac{1}{2} k^{3}\right)\right)_{\sigma \sigma}, \quad \text { in } \mathbb{R} \times\left[0, T_{0}\right], \\
\operatorname{div} \mathbb{C} E(u)=0 \quad \text { in } \Omega_{h}, \\
\mathbb{C} E(u)[\nu]=0 \quad \text { on } \Gamma_{h}, \quad u(x, 0, t)=e_{0}(x, 0), \\
h(\cdot, t) \text { and } \nabla u(\cdot, t) \quad \text { are } b \text {-periodic } \\
h(\cdot, 0)=h_{0}
\end{array}\right.
$$

where $e_{0}$ is a nonzero constant that reflects the mismatch between the crystalline lattices of the film and the substrate and, we recall, $h: \mathbb{R} \times\left[0, T_{0}\right] \rightarrow(0,+\infty)$ denotes the one-dimensional function describing the profile $\Gamma_{h}$ of the film,

$$
J:=\sqrt{1+\left|\frac{\partial h}{\partial x}\right|^{2}}, \quad k:=-\frac{\partial}{\partial x}\left(\frac{\frac{\partial h}{\partial x}}{\sqrt{1+\left|\frac{\partial h}{\partial x}\right|^{2}}}\right)
$$

$Q(E(u))=\frac{1}{2} \mathbb{C} E(u): E(u)$, and $h_{0} \in H_{\text {loc }}^{2}(\mathbb{R})$ is a $b$-periodic function.

As observed by Cahn and Taylor in [12], this motion can be regarded as the $H^{-1}$-gradient flow for the total energy

$$
G(h):=\int_{\Omega_{h}} Q\left(E\left(u_{h}\right)\right) d z+\int_{\Gamma_{h}}\left(g(\theta)+\frac{\varepsilon}{2} k^{2}\right) d \mathcal{H}^{1},
$$


where $\Omega_{h}:=\{(x, y): 0<x<b, 0<y<h(x)\}, \Gamma_{h}$ is the graph of $h$ over the periodicity interval $(0, b)$, and $u_{h}$ is the minimizer of the elastic energy in $\Omega_{h}$ under suitable boundary and periodicity conditions. Therefore, it is natural to adopt De Giorgi's minimizing movements approach (see [4]), which consists in constructing discrete time evolutions by solving iteratively suitable minimum incremental problems. Precisely, we start with a $b$-periodic initial datum $h_{0} \in H_{l o c}^{2}(\mathbb{R})$ and, given $T>0, N \in \mathbb{N}$, for $i=1, \ldots, N$, we define inductively $h_{i, N}$ as the minimizer of

$$
G(h)+\frac{1}{2 \tau} d^{2}\left(h, h_{i-1, N}\right),
$$

where $\tau:=\frac{T}{N}$ and $d$ is a suitable term measuring the $H^{-1}$-distance between $h$ and $h_{i-1, N}$. We mention here that minimizing movements have been already successfully implemented to treat various mean curvature type flows without surface diffusion (see, e.g., $[3,13,8]$ ).

This paper is organized as follows. In Section 2 we set up the problem and introduce the discrete time evolutions. In Section 3 we show that they converge to a weak solution of (1.7) in $\left[0, T_{0}\right]$ for some $T_{0}>0$ (see Theorem 3.8). Precisely, a b-periodic weak solution of (1.7) is a function $h \in H^{1}\left(0, T_{0} ; H_{l o c}^{-1}(\mathbb{R})\right) \cap L^{\infty}\left(0, T_{0} ; H_{l o c}^{2}(\mathbb{R})\right)$, such that $h(\cdot, t)$ is $b$-periodic for all $t \in[0, T]$ and $(h, u)$ satisfies (1.7) in the distributional sense (see Definition 3.1). We remark that Theorem 3.8 seems to be the first (short time) existence result for a surface diffusion type geometric evolution equation in the presence of elasticity. Moreover, also the use of minimizing movements instead of the more classical semigroup approach appears to be new in this context. We observe that in the case without elasticity and without curvature regularization, short time existence of a smooth solution was proved in [17], using semigroup techniques, for the motion of immersed hypersurfaces by surface diffusion. See also [16, 29, 9] and the work of Chen ([14]) for the Hele-Shaw equation.

A delicate point in the proof of our existence result is the choice of $d$ in the penalization term in (1.8) (see Remark 2.2). A rather technical obstacle is overcome in Theorem 3.4, where it is proved that the solutions of the discrete time evolutions are equicontinuous in time with values in $C^{1, \alpha}$ for all $\alpha \in\left(0, \frac{1}{2}\right)$, at least for an initial time interval $\left[0, T_{0}\right]$. This property is crucial to guarantee that the evolving graphs do not develop vertical parts in $\left[0, T_{0}\right]$. However, our variational procedure provides a global in time volume preserving evolution, which satisfies (1.7) until the vertical parts appear (see Theorem 3.3). The main existence result is established in Theorem 3.8.

In Section 4 we prove that the constructed solution $h$ solves the equation in a much stronger sense. Namely, we show that $h \in L^{2}\left(0, T_{0} ; H_{\text {loc }}^{6}(\mathbb{R})\right)$ and $\frac{\partial h}{\partial t} \in L^{2}\left(0, T_{0} ; L_{\text {loc }}^{2}(\mathbb{R})\right)$, provided that the anisotropy $\psi$ is sufficiently smooth (see Theorem 4.3). We note that this is obtained without requiring any further regularity on the initial datum, besides $H_{l o c}^{2}(\mathbb{R})$. The presence of the elasticity term poses some serious technical difficulties in the proof of Theorem 4.3. To understand why, recall that by the classical elliptic theory if the profile $h \in C^{k, \alpha}$ for $k \in \mathbb{N}$ and $\alpha \in(0,1)$, then the corresponding elastic equilibrium $u_{h}$ is of class $C^{k, \alpha}$ up to $\Gamma_{h}$. However, in order to prove the desired regularity result we need to specify in a rather precise way how the constants in the elliptic estimates depend on $h$. This is achieved in Theorem 4.1, whose technical proof makes an essential use of the Airy functions associated to $u_{h}$.

Finally, in Section 5 we show that weak solutions to equation (1.7) are unique and thus coincide necessarily with the solution constructed via minimizing movements.

Future work will address other properties such as long time existence and asymptotic stability.

\section{Setting of the PROBlem}

In this section we introduce the precise mathematical setting needed to define the free-energy functional and the corresponding minimum incremental problems. Our formulation is similar to the one in [10] (see also [21]) to model the epitaxial growth of an elastic film over a rigid substrate 
in the presence of a mismatch between the lattices of the two materials. Following the physical literature and as in [10], we work under periodicity conditions on the evolving profiles and on the corresponding elastic displacements. Given a positive $b$-periodic function $h: \mathbb{R} \rightarrow[0,+\infty)$, with locally finite pointwise variation, we set

$$
\begin{gathered}
\Omega_{h}:=\{z=(x, y): 0<x<b, 0<y<h(x)\}, \quad \Omega_{h}^{\#}:=\{z=(x, y): x \in \mathbb{R}, 0<y<h(x)\} \\
\Gamma_{h}:=\left\{z=(x, y): x \in[0, b), h^{-}(x) \leq y \leq h^{+}(x)\right\}
\end{gathered}
$$

where

$$
h^{-}(x):=\min \{h(x-), h(x+)\} \quad \text { and } \quad h^{+}(x):=\max \{h(x-), h(x+)\},
$$

with $h(x \pm)$ the right and left limit of $h$ at $x$, respectively. We also consider the set

$$
\Gamma_{h}^{\#}:=\left\{z=(x, y): x \in \mathbb{R}, h^{-}(x) \leq y \leq h^{+}(x)\right\} .
$$

The set $\Omega_{h}$ represents the reference configuration of the film over the interval $(0, b)$ and $\Gamma_{h}$ is the corresponding profile. We introduce the class of admissible profile functions $A P$, defined as

$A P:=\{h: \mathbb{R} \rightarrow[0,+\infty): h b$-periodic, lower semicontinuous, $\operatorname{Var}(h ; 0, b)<+\infty$, and there exists

$$
\left.\gamma \in H_{\mathrm{loc}}^{2}\left(\mathbb{R} ; \mathbb{R}^{2}\right) \text { such that } \gamma(t+1)=\gamma(t)+(b, 0),|\dot{\gamma}| \equiv \mathcal{H}^{1}\left(\Gamma_{h}\right) \text {, and } \gamma(\mathbb{R})=\Gamma_{h}^{\#}\right\} \text {. }
$$

Given $h \in A P$, we denote

$L D_{\#}\left(\Omega_{h} ; \mathbb{R}^{2}\right):=\left\{u \in L_{\mathrm{loc}}^{2}\left(\Omega_{h}^{\#} ; \mathbb{R}^{2}\right): u(x, y)=u(x+b, y)\right.$ for $\left.(x, y) \in \Omega_{h}^{\#},\left.E(u)\right|_{\Omega_{h}} \in L^{2}\left(\Omega_{h} ; \mathbb{R}^{2}\right)\right\}$, where $E(u):=\frac{1}{2}\left(\nabla u+\nabla^{T} u\right), \nabla u$ is the distributional gradient of $u$ and $\nabla^{T} u$ is the transpose of $\nabla u$. We work within the theory of small deformations, so that $E(u)$ represents the strain and $u$ is the planar displacement. We also prescribe the Dirichlet boundary condition $u(x, 0)=e_{0}(x, 0)$ at the interface between film and substrate, which models the case of a film growing on an infinitely rigid substrate. This boundary condition forces the film to be strained, thus generating elastic energy. The positive constant $e_{0}$ measures the mismatch between the lattices of the two materials. Thus, we define

$$
\begin{aligned}
X_{e_{0}}:=\left\{(h, u): h \in A P, u: \Omega_{h}^{\#} \rightarrow \mathbb{R}^{2} \text { s.t. } u(\cdot, \cdot)-e_{0}(\cdot, 0) \in L D_{\#}\left(\Omega_{h} ; \mathbb{R}^{2}\right)\right. & \\
& \text { and } \left.u(x, 0)=\left(e_{0} x, 0\right) \text { for all } x \in \mathbb{R}\right\} .
\end{aligned}
$$

The elastic energy density $Q: \mathbb{M}_{\text {sym }}^{2 \times 2} \rightarrow[0,+\infty)$ takes the form

$$
Q(A):=\frac{1}{2} \mathbb{C} A: A
$$

with $\mathbb{C}$ a fourth-order tensor. We assume that $Q(A)>0$ for all $A \in \mathbb{M}_{\text {sym }}^{2 \times 2} \backslash\{0\}$.

Let $\psi: \mathbb{R}^{2} \rightarrow[0,+\infty)$ be a positively one-homogeneous function of class $C^{2}$ away from the origin. Note that, in particular,

$$
c_{1}|\xi| \leq \psi(\xi) \leq c_{2}|\xi| \quad \text { for all } \xi \in \mathbb{R}^{2},
$$

for some $c_{1}, c_{2}>0$. We are ready to introduce the energy functional. For all $(h, u) \in X_{e_{0}}$ we set

$$
F(h, u):=\int_{\Omega_{h}} Q(E(u)) d z+\int_{\Gamma_{h}}\left(\psi(\nu)+\frac{\varepsilon}{2} k^{2}\right) d \mathcal{H}^{1},
$$

where $k$ denotes the curvature of $\Gamma_{h}, \nu$ is the outer unit normal to $\Omega_{h}$, and $\varepsilon$ is a (small) positive constant. 
Remark 2.1. Note that if $\gamma$ is any constant speed parametrization of $\Gamma_{h}^{\#}$ as in (2.1), then by periodicity we have

$$
\int_{\Gamma_{h}}\left(\psi(\nu)+\frac{\varepsilon}{2} k^{2}\right) d \mathcal{H}^{1}=\int_{\gamma(I)}\left(\psi(\nu)+\frac{\varepsilon}{2} k^{2}\right) d \mathcal{H}^{1}
$$

for every interval $I \subset \mathbb{R}$ of length one.

2.1. The incremental minimum problem. In this subsection we introduce the incremental minimum problems used to define the discrete time evolutions. As standing assumptions throughout this paper, we start from an initial configuration $\left(h_{0}, u_{0}\right) \in X_{e_{0}}$, such that

$$
h_{0} \in H_{\#}^{2}([0, b]), \quad h_{0}>0,
$$

and $u_{0}$ minimizes the elastic energy in $\Omega_{h_{0}}$ among all $u$ with $\left(h_{0}, u\right) \in X_{e_{0}}$. Here, and in what follows, we denote by $H_{\#}^{k}(0, l)$ the space of all functions in $H_{l o c}^{k}(\mathbb{R})$ that are $l$-periodic, endowed with the norm of $H^{k}(0, l)$. A similar convention will be used also for other functional spaces. Also, $H_{\#}^{-1}(0, l)$ stands for the dual space of $H_{\#}^{1}(0, l)$.

Given $T>0, N \in \mathbb{N}$, we set $\Delta T:=\frac{T}{N}$. For $i=1, \ldots, N$ we define inductively $\left(h_{i, N}, u_{i, N}\right)$ as the solution of the minimum problem

$$
\begin{gathered}
\min \left\{F(h, u)+\frac{1}{2 \Delta T} \int_{\Gamma_{h_{i-1, N}}}\left(\int_{0}^{x}\left(h(\zeta)-h_{i-1, N}(\zeta)\right) d \zeta\right)^{2} d \mathcal{H}^{1}(x, y):(h, u) \in X_{e_{0}}\right. \\
\left.\int_{0}^{b} h d x=\int_{0}^{b} h_{0} d x, \int_{\Gamma_{h_{i-1, N}}} \int_{0}^{x}\left(h(\zeta)-h_{i-1, N}(\zeta)\right) d \zeta d \mathcal{H}^{1}(x, y)=0\right\}
\end{gathered}
$$

Then for $x \in \mathbb{R}$ and $(i-1) \Delta T \leq t \leq i \Delta T, i=1, \ldots, N$, we define

$$
h_{N}(x, t):=h_{i-1, N}(x)+\frac{1}{\Delta T}(t-(i-1) \Delta T)\left(h_{i, N}(x)-h_{i-1, N}(x)\right)
$$

and we let $u_{N}(\cdot, t)$ be the elastic equilibrium corresponding to $h_{N}(\cdot, t)$, i.e., the minimizer of the elastic energy in $\Omega_{h_{N}(\cdot, t)}$ among all $u$ such that $\left(h_{N}(\cdot, t), u\right) \in X_{e_{0}}$. We also denote by $\gamma_{i, N}$ and $\gamma_{0}$ admissible constant speed parametrizations of $\Gamma_{h_{i, N}}^{\#}$ and $\Gamma_{h_{0}}^{\#}$, respectively, as in (2.1) and oriented in such a way that $\dot{\gamma}_{i, N} \cdot e_{1}, \dot{\gamma}_{0} \cdot e_{1} \geq 0$.

Remark 2.2 (Interpretation of the penalization term). We remark that the penalization term in (2.5) coincides with the square of the $H^{-1}\left(\Gamma_{i-1, N}\right)$-norm of $f:=\frac{h-h_{i-1, N}}{\sqrt{1+h_{i-1, N}^{\prime 2}}}$, provided $h$ satisfies the constraint in (2.5) and $h_{i-1, N}$ is of class $C^{1}$. Indeed, writing $\Gamma$ instead of $\Gamma_{i-1, N}$, the penalization and the constraints on $h$ in $(2.5)$ reduce to

$$
\int_{\Gamma}\left(\int_{\Gamma\left(z_{0}, z\right)} f(w) d \mathcal{H}^{1}(w)\right)^{2} d \mathcal{H}^{1}(z)
$$

and

$$
\int_{\Gamma} f d \mathcal{H}^{1}=0, \quad \int_{\Gamma} \int_{\Gamma\left(z_{0}, z\right)} f(w) d \mathcal{H}^{1}(w) d \mathcal{H}^{1}(z)=0,
$$

where $z_{0}=\left(0, h_{i-1, N}(0)\right)$ and $\Gamma\left(z_{0}, z\right)$ is the arc of $\Gamma$ connecting $z_{0}$ and $z$. Note that if $f$ is a function on $\Gamma$ satisfying the constraints (2.8), then (2.7) reduces to $\|f\|_{H^{-1}(\Gamma)}^{2}$, once we define

$$
\|\varphi\|_{H^{1}(\Gamma)}^{2}:=\left|\varphi\left(z_{0}\right)\right|^{2}+\int_{\Gamma} \varphi_{\sigma}^{2}(z) d \mathcal{H}^{1}(z)
$$

for every $\varphi \in H^{1}(\Gamma)$, where, we recall, $(\cdot)_{\sigma}$ stands for the tangential derivative along $\Gamma$. In fact, integrating by parts, we obtain

$$
\|f\|_{H^{-1}(\Gamma)}=\sup _{\|\varphi\|_{H^{1}(\Gamma)}=1} \int_{\Gamma} f \varphi d \mathcal{H}^{1}=\sup _{\|\varphi\|_{H^{1}(\Gamma)}=1}\left[-\int_{\Gamma} F \varphi_{\sigma} d \mathcal{H}^{1}+\varphi\left(z_{0}\right) \int_{\Gamma} f d \mathcal{H}^{1}\right]
$$


where $F(z):=\int_{\Gamma\left(z_{0}, z\right)} f(w) d \mathcal{H}^{1}(w)$. Setting $\chi:=\varphi_{\sigma}$ and recalling $(2.9)$, we have

$$
\begin{aligned}
\|f\|_{H^{-1}(\Gamma)}^{2} & =\sup _{0 \leq a \leq 1} \sup _{\substack{\|\chi\|_{L^{2}(\Gamma)}=\sqrt{1-a^{2}} \\
\int_{\Gamma} \chi d \mathcal{H}^{1}=0}}\left[-\int_{\Gamma} F \chi d \mathcal{H}^{1}+a \int_{\Gamma} f d \mathcal{H}^{1}\right]^{2} \\
& =\sup _{0 \leq a \leq 1}\left[\sqrt{1-a^{2}}\left(\int_{\Gamma}\left(F(z)-f_{\Gamma} F d \mathcal{H}^{1}\right)^{2} d \mathcal{H}^{1}(z)\right)^{\frac{1}{2}}+a \int_{\Gamma} f d \mathcal{H}^{1}\right]^{2} \\
& =\int_{\Gamma}\left(F(z)-f_{\Gamma} F d \mathcal{H}^{1}\right)^{2} d \mathcal{H}^{1}(z)+\left(\int_{\Gamma} f d \mathcal{H}^{1}\right)^{2} .
\end{aligned}
$$

Recalling (2.8), the right-hand side of the last identity reduces to (2.7).

Note that if $h$ is a solution to the limiting evolution (1.7), then

$$
\int_{0}^{b} h\left(x, t_{1}\right) d x=\int_{0}^{b} h\left(x, t_{2}\right) d x \quad \int_{\Gamma_{h(\cdot, t)}} \int_{0}^{x} \frac{\partial h}{\partial t}(\zeta, t) d \zeta d \mathcal{H}^{1}=0
$$

for $t, t_{1}$, and $t_{2}$ (see (3.4) for the first identity, the second one is proved similarly). This observation justifies the choice the constraints imposed in (2.5).

An alternative formulation of the incremental minimum problem would be

$$
\min \left\{F(h, u)+\frac{1}{2 \Delta T}\left\|\frac{h-h_{i-1, N}}{J_{i-1, N}}\right\|_{H^{-1}\left(\Gamma_{i-1, N}\right)}^{2}:(h, u) \in X_{e_{0}}\right\}
$$

where the $H^{-1}\left(\Gamma_{i-1, N}\right)$-norm is defined as in (2.10) and $J_{i-1, N}$ denotes the length factor of $\Gamma_{i-1, N}$. This should lead to the same limiting evolution equation, although we shall not pursue this approach here.

The remaining of this subsection is devoted to the proof of the existence of a minimizer for the minimum incremental problem (2.5). We start with a compactness lemma.

Lemma 2.3. Let $\left\{h_{n}\right\} \subset A P$ be such that

$$
\sup _{n}\left\|\gamma_{n}\right\|_{H^{2}\left(0,1 ; \mathbb{R}^{2}\right)}<+\infty
$$

where $\gamma_{n}$ is a constant speed parametrization of $\Gamma_{h_{n}}^{\#}$ as in (2.1). Then, there exists $h \in A P$ such that, up to a subsequence,

(i) $h_{n} \rightarrow h$ in $L^{1}(0, b)$,

(ii) $\Gamma_{h_{n}}^{\#} \rightarrow \Gamma_{h}^{\#}$ in the Hausdorff metric;

(iii) $\mathbb{R}_{+}^{2} \backslash \Omega_{h_{n}}^{\#} \rightarrow \mathbb{R}_{+}^{2} \backslash \Omega_{h}^{\#}$ in the Hausdorff metric;

(iv) $\gamma_{n} \rightarrow \gamma$ weakly in $H^{2}\left(0,1 ; \mathbb{R}^{2}\right)$, where $\gamma$ is a constant speed parametrization of $\Gamma_{h}^{\#}$ as in (2.1).

Proof. For simplicity we set $\Gamma_{n}^{\#}:=\Gamma_{h_{n}}^{\#}$. From (2.11) we have that $\sup _{n} \operatorname{Var}\left(h_{n} ; 0, b\right)<+\infty$. Hence, by the Helly theorem, and up to a (not relabeled) subsequence, we may conclude that there exists a $b$-periodic function $h$ with locally finite pointwise variation such that $h_{n} \rightarrow h$ in $L_{l o c}^{1}(\mathbb{R})$ and pointwise everywhere. Moreover, again by (2.11), we may also assume that $\gamma_{n} \rightarrow \gamma$ weakly in $H^{2}\left(0,1 ; \mathbb{R}^{2}\right)$. Finally, by the Blaschke Compactness theorem (see [5, Theorem 6.1]), and using the periodicity of $\Gamma_{n}^{\#}$, we may also assume that there exists a closed set $\Gamma^{\#}$ such that $\Gamma_{n}^{\#} \rightarrow \Gamma^{\#}$ in the Hausdorff metric. Since $\dot{\gamma}_{n} \rightarrow \dot{\gamma}$ uniformly and $\left|\dot{\gamma}_{n}\right| \equiv \mathcal{H}^{1}\left(\gamma_{n}([0,1])\right)$, we get that $|\dot{\gamma}| \equiv \mathcal{H}^{1}(\gamma([0,1]))$. Moreover, since $\gamma_{n} \rightarrow \gamma$ uniformly, using the definition of Hausdorff convergence, we conclude that $\gamma$ is a parametrization of $\Gamma^{\#}$. 
We now show that $\Gamma^{\#}=\Gamma_{h}^{\#}$. Arguing as in the proof of $\left[19\right.$, Lemma 2.5], we get that $\Gamma_{h}^{\#} \subset \Gamma^{\#}$ and the vertical section $\Gamma_{x}^{\#}:=\left\{t \in \mathbb{R}:(x, t) \in \Gamma^{\#}\right\}$ is a closed interval for all $x \in \mathbb{R}$. Assume by contradiction that $\Gamma_{x}^{\#} \supseteqq\left[h^{-}(x), h^{+}(x)\right]$ and, without loss of generality, that $\Gamma_{x}^{\#}=\left[y_{1}, y_{2}\right]$, with $y_{1} \leq h^{-}(x)$ and $y_{2}>h^{+}(x)$. Let $s \in(0,1)$ such that $\gamma(s)=\left(x, y_{2}\right)$. We claim that there exists $\delta>0$ such that $\gamma(t) \in\{x\} \times \Gamma_{x}^{\#}$ for all $t \in(s-\delta, s+\delta)$. Indeed, otherwise there would exist a sequence $t_{n} \rightarrow s$ such that $\gamma\left(t_{n}\right) \notin\{x\} \times \Gamma_{x}^{\#}$. Since $\gamma\left(t_{n}\right)=\left(x_{n}, y_{n}\right) \rightarrow\left(x, y_{2}\right)$ and $h$ is lower semicontinuous, we would have $x_{n} \neq x$ and $y_{n}-h^{+}\left(x_{n}\right)>\frac{y_{2}-h^{+}(x)}{2}$ for $n$ large. This would imply that $\Gamma^{\#}$ contains infinitely many vertical segments of length greater than $\frac{y_{2}-h^{+}(x)}{2}$ near $\left(x, y_{2}\right)$, which is in contradiction with the fact that $\Gamma^{\#}$ has locally finite length. Hence, writing $\gamma(t)=$ $(x(t), y(t))$, we have that in the interval $(s-\delta, s+\delta), x(t)=x$, and $y(s)=y_{2}=\max _{t \in(s-\delta, s+\delta)} y(t)$. It follows that $\dot{\gamma}(s)=0$, which is impossible. This shows that $\Gamma^{\#}=\Gamma_{h}^{\#}$ and that $\gamma$ is a constant speed parametrization of $\Gamma_{h}^{\#}$ as in $(2.1)$.

To conclude the proof, we recall that under our assumptions there exists (see [10], [21, Theorem 2.2]) a lower semicontinuous $b$-periodic function $\tilde{h}$, with locally finite pointwise variation, such that, up to a subsequence,

$$
\mathbb{R}_{+}^{2} \backslash \Omega_{h_{n}}^{\#} \rightarrow \mathbb{R}_{+}^{2} \backslash \Omega_{\tilde{h}}^{\#} \quad \text { in the Hausdorff metric }
$$

Moreover (see [19, Proposition 2.2]), for all $x \in \mathbb{R}$

$$
\tilde{h}(x)=\inf \left\{\liminf h_{n}\left(x_{n}\right): x_{n} \rightarrow x\right\}
$$

and (see [19, Lemma 2.5]) $\tilde{h}=h$ almost everywhere. Define

$$
\widetilde{\Gamma}_{\tilde{h}}^{\#}:=\left\{(x, y) \in \mathbb{R}^{2}: x \in \mathbb{R}, \tilde{h}(x) \leq y \leq \tilde{h}^{+}(x)=h^{+}(x)\right\},
$$

which is the union of the extended graph $\Gamma_{\tilde{h}}^{\#}=\Gamma_{h}^{\#}$ with the vertical segments of the type $\{x\} \times$ $\left[\tilde{h}(x), \tilde{h}^{-}(x)\right]$.

From (2.12) it follows that $\widetilde{\Gamma}_{\tilde{h}}^{\#}$ is contained in the Hausdorff limit of $\Gamma_{h_{n}}^{\#}$. Hence, by (ii) $\widetilde{\Gamma}_{\tilde{h}}^{\#} \subset \Gamma_{h}^{\#}$. Since the opposite inclusion is obvious, we conclude that the two sets coincide and, in turn, $\Omega_{\tilde{h}}=\Omega_{h}$. This concludes the proof of the lemma.

Using this compactness lemma, we now show that the incremental minimum problem (2.5) admits a solution.

Theorem 2.4. For every $i=1, \ldots, N$, the minimum problem $(2.5)$ admits a solution $\left(h_{i, N}, u_{i, N}\right) \in$ $X_{e_{0}}$.

Proof. Let $\left(h_{n}, u_{n}\right) \in X_{e_{0}}$ be a minimizing sequence for (2.5) and let $\gamma_{n}$ be an admissible constant speed parametrization of $\Gamma_{h_{n}}^{\#}$. Then,

$$
\sup _{n}\left\{F\left(h_{n}, u_{n}\right)+\frac{1}{2 \Delta T} \int_{\Gamma_{h_{i-1}, N}}\left(\int_{0}^{x}\left(h_{n}(\zeta)-h_{i-1, N}(\zeta)\right) d \zeta\right)^{2} d \mathcal{H}^{1}(x, y)\right\}<+\infty
$$

Since by (2.5) and by (2.13) the functions $h_{n}$ are bounded in $B V(0, b)$, we have

$$
\sup _{n}\left\|h_{n}\right\|_{L^{\infty}(0, b)} \leq C .
$$

Moreover, again by (2.13) and by (2.2), we have that

$$
\sup _{n} \int_{0}^{1}\left(\left|\dot{\gamma}_{n}\right|^{2}+\left|\ddot{\gamma}_{n}\right|^{2}\right) d t<+\infty
$$


This, together with (2.14), yields (2.11). Thus we are in a position to apply Lemma 2.3 to conclude that there exists $h \in A P$ such that, for a not relabeled subsequence, (i)-(iv) of that lemma hold. In particular, using (i) and (iv), we have that

$$
\int_{\Gamma_{h}}\left(\psi(\nu)+\frac{\varepsilon}{2} k^{2}\right) d \mathcal{H}^{1} \leq \liminf _{n} \int_{\Gamma_{h_{n}}}\left(\psi(\nu)+\frac{\varepsilon}{2} k^{2}\right) d \mathcal{H}^{1}
$$

and

$$
\int_{\Gamma_{h_{i-1, N}}}\left(\int_{0}^{x}\left(h(\zeta)-h_{i-1, N}(\zeta)\right) d \zeta\right)^{2} d \mathcal{H}^{1}=\lim _{n} \int_{\Gamma_{h_{i-1, N}}}\left(\int_{0}^{x}\left(h_{n}(\zeta)-h_{i-1, N}(\zeta)\right) d \zeta\right)^{2} d \mathcal{H}^{1}
$$

Finally, since $\sup _{n} \int_{\Omega_{h_{n}}}\left|E u_{n}\right|^{2} d z<+\infty$, reasoning as in [19, Proposition 2.2], from (iii) and Korn's inequality we conclude that there exists $u \in H_{l o c}^{1}\left(\Omega_{h}^{\#} ; \mathbb{R}^{2}\right)$ such that $(h, u) \in X_{e_{0}}$ and, up to a subsequence, $u_{n} \rightarrow u$ weakly in $H_{l o c}^{1}\left(\Omega_{h}^{\#} ; \mathbb{R}^{2}\right)$. Therefore, we have that

$$
\int_{\Omega_{h}} Q(E(u)) d z \leq \liminf _{n} \int_{\Omega_{h_{n}}} Q\left(E\left(u_{n}\right)\right) d z,
$$

which, together with (2.15) and (2.16), allows us to conclude that $(h, u)$ is a minimizer.

\section{Existence OF the EVOLUtion}

In this section we show that solutions of the discrete time evolution problems defined in the previous section (see (2.6)) converge to a function $h=h(x, t)$ such that $\Gamma_{h(\cdot, t)}$ is a suitable weak solution (see Definition 3.1 below) of the following geometric evolution equation,

$$
V=\left(\left(g_{\theta \theta}+g\right) k+Q(E(u))-\varepsilon\left(k_{\sigma \sigma}+\frac{1}{2} k^{3}\right)\right)_{\sigma \sigma}
$$

provided that the initial configuration $\left(h_{0}, u_{0}\right) \in X_{e_{0}}$ satisfies (2.4). Here $V$ denotes the outer normal velocity of $\Gamma_{h(\cdot, t)}, k$ is its curvature, $Q(E(u))$ is the trace of $Q(E(u(\cdot, t)))$ on $\Gamma_{h(\cdot, t)}$, with $u(\cdot, t)$ the elastic equilibrium in $\Omega_{h(\cdot, t)}$. Moreover, $g:[0,2 \pi] \rightarrow(0,+\infty)$ is defined as

$$
g(\theta)=\psi(\cos \theta, \sin \theta)
$$

and is evaluated at $\arg (\nu(\cdot, t))$, where $\nu(\cdot, t)$ is the outer normal to $\Gamma_{h(\cdot, t)}$.

As in Section 2, fix T, N, and define $h_{N}$ as in (2.6). Throughout this section we will assume that the initial profile $h_{0}$ belongs to $H_{\#}^{2}(0, b)$ and is strictly positive. A function $f \in L^{2}\left(\Gamma_{h}\right)$ will be identified with the functional:

$$
\varphi \in H_{\#}^{1}(0, b) \mapsto\langle f, \varphi\rangle:=\int_{0}^{b} f \varphi d x
$$

We now introduce a suitable notion of weak solution of equation (3.1).

Definition 3.1. We say that $h \in H^{1}\left(0, T_{0} ; H_{\#}^{-1}(0, b)\right) \cap L^{\infty}\left(0, T_{0} ; H_{\#}^{2}(0, b)\right)$ is a weak solution of (3.1) in $\left[0, T_{0}\right]$ if:

(i) for almost every $t \in\left[0, T_{0}\right]$ the function $h(\cdot, t) \in A P \cap H_{\#}^{4}(0, b)$;

(ii) $\left(g_{\theta \theta}+g\right) k+Q(E(u))-\varepsilon\left(k_{\sigma \sigma}+\frac{1}{2} k^{3}\right) \in L^{2}\left(0, T_{0} ; H_{\#}^{1}(0, b)\right)$, where $Q(E(u))$ is the trace of $Q(E(u(\cdot, t)))$ on $\Gamma_{h(\cdot, t)}$, and $u(\cdot, t)$ is the elastic equilibrium in $\Omega_{h(\cdot, t)}$;

(iii) for almost every $t \in\left[0, T_{0}\right]$

$$
\frac{\partial h}{\partial t}=J\left(\left(g_{\theta \theta}+g\right) k+Q(E(u))-\varepsilon\left(k_{\sigma \sigma}+\frac{1}{2} k^{3}\right)\right)_{\sigma \sigma} \quad \text { in } H_{\#}^{-1}(0, b) .
$$

Note that in context of the Definition 3.1, $J=\sqrt{1+h_{x}^{2}},(\cdot)_{\sigma}=\frac{1}{J}(\cdot)_{x}$, and the outer normal velocity $V$ coincides with $\frac{1}{J} \frac{\partial h}{\partial t}$. 
Remark 3.2. Concerning the definition above we observe that

(i) from Lemmas 6.6 and 6.7 it follows that

$$
\left(g_{\theta \theta}+g\right) k-\varepsilon\left(k_{\sigma \sigma}+\frac{1}{2} k^{3}\right)=\varepsilon\left(\frac{h_{x x}}{J^{5}}\right)_{x x}+\frac{5 \varepsilon}{2}\left(\frac{h_{x x}^{2}}{J^{7}} h_{x}\right)_{x}+\left(\psi_{x}\left(-h_{x}, 1\right)\right)_{x},
$$

and so

$$
\frac{\partial h}{\partial t}=\left[\frac{1}{J}\left(\varepsilon\left(\frac{h_{x x}}{J^{5}}\right)_{x x}+\frac{5 \varepsilon}{2}\left(\frac{h_{x x}^{2}}{J^{7}} h_{x}\right)_{x}+\left(\psi_{x}\left(-h_{x}, 1\right)\right)_{x}\right)_{x}\right]_{x} .
$$

(ii) Another immediate consequence of the above definition is that the evolution is volume preserving, that is, $\int_{0}^{b} h(x, t) d x=\int_{0}^{b} h_{0}(x) d x$ for all $t \in\left[0, T_{0}\right]$. Indeed, for all $t_{1}, t_{2} \in\left[0, T_{0}\right]$ and for $\varphi \in H_{\#}^{1}(0, b)$ we have

$$
\begin{aligned}
\int_{0}^{b}\left[h\left(x, t_{2}\right)\right. & \left.-h\left(x, t_{1}\right)\right] \varphi(x) d x=\int_{t_{1}}^{t_{2}}\left\langle\frac{\partial h}{\partial t}(\cdot, t), \varphi\right\rangle d t \\
& =\int_{t_{1}}^{t_{2}}\left\langle J\left(\left(g_{\theta \theta}+g\right) k+Q(E(u))-\varepsilon\left(k_{\sigma \sigma}+\frac{1}{2} k^{3}\right)\right)_{\sigma \sigma}, \varphi\right\rangle d t \\
& =-\int_{t_{1}}^{t_{2}} \int_{0}^{b}\left(\left(g_{\theta \theta}+g\right) k+Q(E(u))-\varepsilon\left(k_{\sigma \sigma}+\frac{1}{2} k^{3}\right)\right)_{\sigma} \varphi^{\prime} d x d t .
\end{aligned}
$$

Choosing $\varphi=1$ we conclude that

$$
\int_{0}^{b} h\left(x, t_{2}\right) d x=\int_{0}^{b} h\left(x, t_{1}\right) d x .
$$

The remaining part of this section is devoted to showing that the functions $h_{N}$ constructed in Section 2 converge to a weak solution of (3.1). We start by proving that the functions $h_{N}$ are uniformly bounded in $H^{1}\left((0, T) ; H_{\#}^{-1}(0, b)\right)$. Precisely, we have

Theorem 3.3. For all $N, i=1, \ldots, N$, and $T$ we have

$$
\begin{gathered}
\int_{0}^{T} \int_{0}^{b}\left(\int_{0}^{x} \frac{\partial h_{N}}{\partial t}(\zeta, t) d \zeta\right)^{2} d x d t \leq 2 F\left(h_{0}, u_{0}\right), \\
F\left(h_{i, N}, u_{i, N}\right) \leq F\left(h_{0}, u_{0}\right)
\end{gathered}
$$

and

$$
\sup _{i, N}\left\|\gamma_{i, N}\right\|_{H^{2}\left(0,1 ; \mathbb{R}^{2}\right)}<+\infty
$$

Moreover, up to a subsequence,

$h_{N} \rightarrow h$ in $C^{0, \alpha}\left([0, T] ; L^{2}(0, b)\right)$ for all $\alpha \in\left(0, \frac{1}{8}\right), \quad h_{N} \rightarrow h$ weakly in $H^{1}\left(0, T ; H_{\#}^{-1}(0, b)\right)$,

for some function $h$ such that $h(\cdot, t) \in A P$ for every $t \in[0, T]$.

Proof. By the minimality of $\left(h_{i, N}, u_{i, N}\right)$ we have that

$$
F\left(h_{i, N}, u_{i, N}\right)+\frac{1}{2 \Delta T} \int_{\Gamma_{h_{i-1, N}}}\left(\int_{0}^{x}\left(h_{i, N}(\zeta)-h_{i-1, N}(\zeta)\right) d \zeta\right)^{2} d \mathcal{H}^{1} \leq F\left(h_{i-1, N}, u_{i-1, N}\right)
$$

for all $i=1, \ldots, N$. Hence,

$$
\frac{1}{2 \Delta T} \int_{0}^{b}\left(\int_{0}^{x}\left(h_{i, N}(\zeta)-h_{i-1, N}(\zeta)\right) d \zeta\right)^{2} d x \leq F\left(h_{i-1, N}, u_{i-1, N}\right)-F\left(h_{i, N}, u_{i, N}\right) .
$$

Summing over $i=1, \ldots, N$, we obtain

$$
\sum_{i=1}^{N} \frac{1}{2 \Delta T} \int_{0}^{b}\left(\int_{0}^{x}\left(h_{i, N}(\zeta)-h_{i-1, N}(\zeta)\right) d \zeta\right)^{2} d x \leq F\left(h_{0}, u_{0}\right)
$$


from which we deduce the estimate (3.5) (see (2.6)).

Moreover, by iterating (3.9) we obtain (3.6) and, in particular, recalling (2.2)

$$
\mathcal{H}^{1}\left(\Gamma_{h_{i, N}}\right) \leq \frac{1}{c_{1}} F\left(h_{0}, u_{0}\right) .
$$

Hence,

$$
\sup _{N, t}\left|D h_{N}(\cdot, t)\right|(0, b) \leq C
$$

for some positive constant $C>0$. By (3.5) we have for $t_{2}>t_{1}$

$$
\begin{aligned}
\left\|h_{N}\left(\cdot, t_{2}\right)-h_{N}\left(\cdot, t_{1}\right)\right\|_{H^{-1}} & \leq \int_{t_{1}}^{t_{2}}\left\|\frac{\partial h_{N}(\cdot, t)}{\partial t}\right\|_{H^{-1}} d t \\
& \leq\left(t_{2}-t_{1}\right)^{\frac{1}{2}}\left(\int_{t_{1}}^{t_{2}}\left\|\frac{\partial h_{N}(\cdot, t)}{\partial t}\right\|_{H^{-1}}^{2} d t\right)^{\frac{1}{2}} \leq C\left(t_{2}-t_{1}\right)^{\frac{1}{2}} .
\end{aligned}
$$

Applying Remark 6.5 to the $f(x)=\int_{0}^{x}\left(h_{N}\left(\zeta, t_{1}\right)-h_{N}\left(\zeta, t_{2}\right)\right) d \zeta$, which vanishes at 0 and $b$ (see $(2.5)$ ), and recalling (3.10), from (3.11) we get

$$
\begin{aligned}
\left\|h_{N}\left(\cdot, t_{2}\right)-h_{N}\left(\cdot, t_{1}\right)\right\|_{L^{2}} & \leq C\left(\left|D\left(h_{N}\left(\cdot, t_{2}\right)-h_{N}\left(\cdot, t_{1}\right)\right)\right|(0, b)\right)^{\frac{3}{4}}\left\|h_{N}\left(\cdot, t_{2}\right)-h_{N}\left(\cdot, t_{1}\right)\right\|_{H^{-1}}^{\frac{1}{4}} \\
& \leq C\left(t_{2}-t_{1}\right)^{\frac{1}{8}} .
\end{aligned}
$$

By the Ascoli-Arzelà theorem (see e.g. [6, Proposition 3.3.1]) we find a subsequence (not relabeled) and a function $h$ such that

$$
h_{N} \rightarrow h \quad \text { in } C^{0, \alpha}\left(0, T ; L^{2}(0, b)\right) \text { for all } \alpha \in\left(0, \frac{1}{8}\right) .
$$

Finally, observe that from (3.6) we have (3.7). Hence, since for every $t \in[0, T]$ we may find a sequence $\left(i_{n}, N_{n}\right)$ such that $h_{i_{n}, N_{n}} \rightarrow h(\cdot, t)$ in $L^{2}(0, b)$, by Lemma 2.3 we conclude that $h(\cdot, t) \in$ $A P$. The weak convergence of $h_{N}$ to $h$ in $H^{1}\left(0, T ; H_{\#}^{-1}(0, b)\right)$ follows from (3.5). This concludes the proof of the theorem.

In the following, $h_{N}$ and $h$ will denote the subsequence and the function provided by Theorem 3.3, respectively. The next result shows that the convergence of $h_{N}$ to $h$ can be significantly improved for short time.

Theorem 3.4. There exist $T_{0} \in(0, T]$ and $C>0$ depending only $\left(h_{0}, u_{0}\right)$ such that:

(i) $h_{N} \rightarrow h$ in $C^{0, \beta}\left(\left[0, T_{0}\right] ; C^{1, \alpha}([0, b])\right)$ for every $\alpha \in\left(0, \frac{1}{2}\right)$, and $\beta \in(0,(1-2 \alpha) / 16)$;

(ii) $\sup _{t \in\left[0, T_{0}\right]}\left\|h_{N}(\cdot, t)\right\|_{H^{2}(0, b)} \leq C$;

(iii) $\sup _{t \in\left[0, T_{0}\right]}\left\|\nabla u_{N}(\cdot, t)\right\|_{C^{0, \frac{1}{2}}\left(\bar{\Omega}_{h_{N}(\cdot, t)}\right)} \leq C$;

(iv) $E\left(u_{N}\left(\cdot, h_{N}\right)\right) \rightarrow E(u(\cdot, h))$ in $C^{0, \beta}\left(\left[0, T_{0}\right] ; C^{1, \alpha}([0, b])\right)$ for every $\alpha \in\left(0, \frac{1}{2}\right)$, and $0 \leq \beta<$ $(1-2 \alpha) / 16$, where $u(\cdot, t)$ is the elastic equilibrium in $\Omega_{h(\cdot, t)}$.

In particular, $h(\cdot, t) \rightarrow h_{0}$ in $C^{1, \alpha}([0, b])$ as $t \rightarrow 0^{+}$and $h_{N}, h \geq C_{0}>0$ for some positive constant $C_{0}$, provided that $N$ is sufficiently large and $T_{0}$ is small enough.

Proof. We claim that for every $\eta \in(0,1)$ there exists $\delta_{\eta}>0$ such that if $\frac{i T}{N} \leq \delta_{\eta}$, then

$$
\inf _{0 \leq \tau \leq 1}\left\|\gamma_{i, N}-\gamma_{0}(\cdot+\tau)\right\|_{L^{2}(0,1)}<\eta,
$$

where, we recall, $\gamma_{0}, \gamma_{i, N}$ are admissible constant speed parametrization of $\Gamma_{h_{0}}^{\#}, \Gamma_{h_{i, N}}^{\#}$, respectively, such that $\dot{\gamma}_{0} \cdot e_{1}, \dot{\gamma}_{i, N} \cdot e_{1} \geq 0$. Indeed, if not there exist $\eta_{0}>0$ and $i_{n}, N_{n}$ such that $\frac{i_{n} T}{N_{n}} \leq \frac{1}{n}$ and

$$
\inf _{0 \leq \tau \leq 1}\left\|\gamma_{i_{n}, N_{n}}-\gamma_{0}(\cdot+\tau)\right\|_{L^{2}(0,1)} \geq \eta_{0}
$$


for all $n \in \mathbb{N}$. Since $h_{i_{n}, N_{n}}=h_{N_{n}}\left(\cdot, \frac{i_{n} T}{N_{n}}\right)$ (see (2.6)), by Theorem 3.3 we have that $h_{i_{n}, N_{n}}$ converge in $L^{2}(0, b)$ to $h_{0}$. Recalling $(3.7)$, by Lemma 2.3 we have that, up to a further (not relabeled) subsequence, $\gamma_{i_{n}, N_{n}} \rightarrow \gamma_{1}$ weakly in $H_{\#}^{2}\left(0,1 ; \mathbb{R}^{2}\right)$, where $\gamma_{1}$ is an admissible constant speed parametrization of $\Gamma_{h_{0}}^{\#}$. Hence, $\gamma_{1}=\gamma_{0}\left(\cdot+\tau_{0}\right)$ for some $0 \leq \tau_{0} \leq 1$. We deduce that

$$
\left\|\gamma_{i_{n}, N_{n}}-\gamma_{0}\left(\cdot+\tau_{0}\right)\right\|_{L^{2}(0,1)} \rightarrow 0,
$$

which is in contradiction with (3.14). This proves the claim.

Let $\tau_{i, N} \in[0,1]$ be such that

$$
\left\|\gamma_{i, N}-\gamma_{0}\left(\cdot+\tau_{i, N}\right)\right\|_{L^{2}(0,1)}<\eta
$$

for $\frac{i T}{N} \leq \delta_{\eta}$. We now apply Theorem 6.4 to $f:=\gamma_{i, N}-\gamma_{0}\left(\cdot+\tau_{i, N}\right)$, to obtain

$$
\begin{aligned}
\left\|\dot{\gamma}_{i, N}-\dot{\gamma}_{0}\left(\cdot+\tau_{i, N}\right)\right\|_{L^{\infty}} \leq & C\left\|\ddot{\gamma}_{i, N}-\ddot{\gamma}_{0}\left(\cdot+\tau_{i, N}\right)\right\|_{L^{2}}^{\frac{3}{4}}\left\|\gamma_{i, N}-\gamma_{0}\left(\cdot+\tau_{i, N}\right)\right\|_{L^{2}}^{\frac{1}{4}} \\
& +C\left\|\gamma_{i, N}-\gamma_{0}\left(\cdot+\tau_{i, N}\right)\right\|_{L^{2}} \leq C \eta^{\frac{1}{4}}
\end{aligned}
$$

where the last inequality follows from (3.7) and (3.13), provided that $\frac{i T}{N} \leq \delta_{\eta}$.

Since $\gamma_{0}(s)=\left(x_{0}(s), y_{0}(s)\right)$ is a constant speed parametrization of a $C^{1}$ graph, we have that $\dot{x}_{0}(s) \geq c_{0}$ for some $c_{0}>0$. Therefore, if $\eta$ is sufficiently small, and writing $\gamma_{i, N}(s)=$ $\left(x_{i, N}(s), y_{i, N}(s)\right)$, we have that $\dot{x}_{i, N}(s) \geq \frac{c_{0}}{2}$. This fact implies in particular that $h_{i, N} \in C^{1}([0, b])$ and

$$
\left\|\frac{h_{i, N}^{\prime}}{\sqrt{1+\left(h_{i, N}^{\prime}\right)^{2}}}-\frac{h_{0}^{\prime}}{\sqrt{1+\left(h_{0}^{\prime}\right)^{2}}}\right\|_{L^{\infty}(0, b)} \leq \omega(\eta),
$$

where $\omega(\eta) \rightarrow 0$ as $\eta \rightarrow 0^{+}$. Let

$$
M:=\max _{x \in[0, b]}\left|\frac{h_{0}^{\prime}(x)}{\sqrt{1+\left(h_{0}^{\prime}(x)\right)^{2}}}\right|<1 .
$$

By taking $\eta$ in (3.15) so small that $M+\omega(\eta)<1$, we obtain

$$
\left\|\frac{h_{i, N}^{\prime}}{\sqrt{1+\left(h_{i, N}^{\prime}\right)^{2}}}\right\|_{L^{\infty}(0, b)} \leq M+\omega(\eta)<1,
$$

for $\frac{i T}{N} \leq \delta_{\eta}=: T_{0}$. Hence, $\left\|h_{i, N}^{\prime}\right\|_{L^{\infty}(0, b)} \leq C$ and, in turn, by (3.7) we get that

$$
\left\|h_{i, N}^{\prime \prime}\right\|_{L^{2}(0, b)} \leq C
$$

for $\frac{i T}{N} \leq T_{0}$ and for some $C>0$. From this estimate, recalling (2.6), assertion (ii) of the statement follows.

To prove assertion (i), we start by observing that by Theorem 6.4, the property (ii) just proved, and (3.12) we have

$$
\begin{aligned}
\left\|\frac{\partial h_{N}}{\partial x}\left(\cdot, t_{2}\right)-\frac{\partial h_{N}}{\partial x}\left(\cdot, t_{1}\right)\right\|_{L^{\infty}} & \leq C\left\|\frac{\partial^{2} h_{N}}{\partial x^{2}}\left(\cdot, t_{2}\right)-\frac{\partial^{2} h_{N}}{\partial x^{2}}\left(\cdot, t_{1}\right)\right\|_{L^{2}}^{\frac{1}{2}}\left\|h_{N}\left(\cdot, t_{2}\right)-h_{N}\left(\cdot, t_{1}\right)\right\|_{L^{2}}^{\frac{1}{2}} \\
& \leq C\left|t_{2}-t_{1}\right|^{\frac{1}{16}}
\end{aligned}
$$

for all $t_{1}, t_{2} \in\left[0, T_{0}\right]$. Notice that from property (ii) we have

$$
\sup _{N, t \in\left[0, T_{0}\right]}\left\|h_{N}(\cdot, t)\right\|_{C^{1, \frac{1}{2}([0, b])}}<+\infty .
$$


Take $\alpha \in\left(0, \frac{1}{2}\right)$ and observe that,

$$
\left|\frac{\partial h_{N}}{\partial x}\left(\cdot, t_{2}\right)-\frac{\partial h_{N}}{\partial x}\left(\cdot, t_{1}\right)\right|_{\alpha} \leq\left|\frac{\partial h_{N}}{\partial x}\left(\cdot, t_{2}\right)-\frac{\partial h_{N}}{\partial x}\left(\cdot, t_{1}\right)\right|_{\frac{1}{2}}^{2 \alpha}\left[\underset{[0, b]}{\operatorname{asc}}\left(\frac{\partial h_{N}}{\partial x}\left(\cdot, t_{2}\right)-\frac{\partial h_{N}}{\partial x}\left(\cdot, t_{1}\right)\right)\right]^{1-2 \alpha},
$$

where $|\cdot|_{\beta}$ denotes the $\beta$-Hölder seminorm. From this inequality, (3.16), and (3.17) assertion (i) follows.

Standard elliptic estimates (see [21, Proprosition 8.9]) ensure that if $h_{N}(\cdot, t) \in C^{1, \alpha}([0, b])$ for some $\alpha \in(0,1)$, then $\nabla u_{N}(\cdot, t)$ can be estimated in $C^{0, \alpha}\left(\bar{\Omega}_{h_{N}(\cdot, t)}\right)$ with a constant depending only on the $C^{1, \alpha}$-norm of $h_{N}(\cdot, t)$. Hence, assertion (iii) follows from (3.17). Assertion (iv) is an immediate consequence of (i) and Lemma 6.10 .

In what follows $J_{i, N}$ stands for

$$
J_{i, N}:=\sqrt{1+\left(h_{i, N}^{\prime}\right)^{2}} .
$$

Theorem 3.5. Let $T_{0}$ and $h_{N}$ be as in Theorem 3.4. Then there exists $C>0$ such that

$$
\int_{0}^{T_{0}} \int_{0}^{b}\left|\frac{\partial^{4} h_{N}}{\partial x^{4}}(x, t)\right|^{2} d x d t \leq C
$$

for $N \in \mathbb{N}$.

Proof. For every $N, i=1, \ldots, N$, the Euler-Lagrange equation satisfied by $h_{i, N}$ is

$$
\int_{0}^{b}\left[\varepsilon \frac{h_{i, N}^{\prime \prime}}{J_{i, N}^{5}} \varphi^{\prime \prime}-\frac{5 \varepsilon}{2} \frac{\left(h_{i, N}^{\prime \prime}\right)^{2}}{J_{i, N}^{7}} h_{i, N}^{\prime} \varphi^{\prime}-\psi_{x}\left(-h_{i, N}^{\prime}, 1\right) \varphi^{\prime}\right] d x+\int_{0}^{b}\left(Q_{i, N}-H_{i, N}\right) \varphi d x=0
$$

for every $\varphi \in H_{\#}^{2}(0, b)$ such that $\int_{0}^{b} \varphi d x=0$ and $\int_{\Gamma_{h_{i-1, N}}} \int_{0}^{x} \varphi d \zeta d \mathcal{H}^{1}=0$, where

$$
\begin{aligned}
& H_{i, N}(x):=\int_{0}^{x}\left(\int_{0}^{\zeta} \frac{h_{i, N}(r)-h_{i-1, N}(r)}{\Delta T} d r\right) J_{i-1, N}(\zeta) d \zeta, \\
& Q_{i, N}(x):=Q\left(E\left(u_{i, N}\left(x, h_{i, N}(x)\right)\right)\right) .
\end{aligned}
$$

We start by showing that $h_{i, N} \in H_{\#}^{4}(0, b)$ and that its fourth derivative, $h_{i, N}^{(\mathrm{iv})}$, is continuous and thus periodic. To see this, note that (3.19) implies that

$$
\int_{0}^{b} f_{i, N} \varphi^{\prime \prime} d x=0
$$

for all $\varphi \in C_{c}^{2}(0, b)$ with $\int_{0}^{b} \varphi d x=0$ and $\int_{\Gamma_{h_{i-1, N}}} \int_{0}^{x} \varphi d \zeta d \mathcal{H}^{1}=0$, where

$$
f_{i, N}(x):=\varepsilon \frac{h_{i, N}^{\prime \prime}}{J_{i, N}^{5}}+\int_{0}^{x}\left(\frac{5 \varepsilon}{2} \frac{\left(h_{i, N}^{\prime \prime}\right)^{2}}{J_{i, N}^{7}} h_{i, N}^{\prime}+\psi_{x}\left(-h_{i, N}^{\prime}, 1\right)\right) d r+\int_{0}^{x} \int_{0}^{r}\left[Q_{i, N}-H_{i, N}\right] d \zeta d r
$$

is an $L^{2}$-function. Hence, by Lemma 6.8 we get that

$$
f_{i, N}(x)=a_{i, N} \int_{0}^{x} \int_{0}^{x_{2}} \int_{0}^{x_{1}} J_{i-1, N} d r d x_{1} d x_{2}+c_{i, N} x^{2}+d_{i, N} x+e_{i, N}
$$

for some constants $a_{i, N}, c_{i, N}, d_{i, N}$, and $e_{i, N}$. Using (3.21) and (3.22), we may conclude that $h_{i, N}^{\prime \prime} \in W^{1,1}(0, b)$. By a bootstrap argument, using again (3.21), (3.22), standard elliptic estimates, and the fact $\psi \in C^{2}\left(\mathbb{R}^{2} \backslash\{0\}\right)$, we get that $h_{i, N} \in H^{4}(0, b)$ and $h_{i, N}^{(\mathrm{iv})}$ is continuous in $(0, b)$. 
It remains to prove periodicity. To this purpose, note that, given any $a \in \mathbb{R}$, for every $i=1, \ldots, N, h_{i, N}$ is also a minimizer of the functional

$$
\begin{aligned}
\int_{a}^{a+b} \int_{0}^{h(x)} Q(E(u)) d y d x & +\int_{a}^{a+b}\left(\psi(\nu)+\frac{\varepsilon}{2} k^{2}\right) J d x \\
& +\frac{1}{2 \Delta T} \int_{a}^{a+b}\left(\int_{0}^{x}\left(h(\zeta)-h_{i-1, N}(\zeta)\right) d \zeta\right)^{2} J_{i-1, N} d x
\end{aligned}
$$

among all $(h, u) \in X_{e_{0}}$ such that $h \in H_{\#}^{2}(0, b), \int_{0}^{b} h d x=\int_{0}^{b} h_{0} d x$, and $\int_{\Gamma_{h_{i-1, N}}} \int_{0}^{x}(h(\zeta)-$ $\left.h_{i-1, N}(\zeta)\right) d \zeta d \mathcal{H}^{1}=0$. This is a consequence of the translation invariance of the energy, which in turn follows from the $b$-periodicity of $h, h_{i-1, N}$, and $x \mapsto \int_{0}^{x}\left(h-h_{i-1, N}\right) d \zeta$ (see (2.5)). Writing the Euler-Lagrange equation satisfied by $h_{i, N}$ in the interval $(a, a+b)$, we get

$$
\int_{a}^{a+b}\left[\varepsilon \frac{h_{i, N}^{\prime \prime}}{J_{i, N}^{5}} \varphi^{\prime \prime}-\frac{5 \varepsilon}{2} \frac{\left(h_{i, N}^{\prime \prime}\right)^{2}}{J_{i, N}^{7}} h_{i, N}^{\prime} \varphi^{\prime}-\psi_{x}\left(-h_{i, N}^{\prime}, 1\right) \varphi^{\prime}\right] d x+\int_{a}^{a+b}\left(Q_{i, N}-H_{i, N}\right) \varphi d x=0,
$$

where we have used the fact that

$$
\begin{aligned}
& \frac{d}{d \eta}\left[\frac{1}{2 \Delta T} \int_{a}^{a+b}\left(\int_{0}^{x}\left(h_{i, N}(\zeta)+\eta \varphi(\zeta)-h_{i-1, N}(\zeta)\right) d \zeta\right)^{2} J_{i-1, N} d x\right]_{\left.\right|_{\eta=0}} \\
& =\int_{a}^{a+b}\left[\int_{0}^{x} \varphi d \zeta \int_{0}^{x} \frac{h_{i, N}-h_{i-1, N}}{\Delta T} d \zeta\right] J_{i-1, N} d x \\
& =H_{i, N}(a+b) \int_{0}^{a+b} \varphi d \zeta-H_{i, N}(a) \int_{0}^{a} \varphi d \zeta-\int_{a}^{a+b} \varphi H_{i, N} d x \\
& =\left(H_{i, N}(a+b)-H_{i, N}(a)\right) \int_{0}^{a} \varphi d \zeta-\int_{a}^{a+b} \varphi H_{i, N} d x \\
& =-\int_{a}^{a+b} \varphi H_{i, N} d x .
\end{aligned}
$$

Observe that the second equality follows by integrating by parts, while the last two follow from the fact that $\int_{a}^{a+b} \varphi d \zeta=0$ and the periodicity of $H_{i, N}$, respectively.

Now arguing as before in any interval of the form $(a, a+b)$ and using equation (3.23), we deduce that $h_{i, N} \in H_{l o c}^{4}(\mathbb{R})$ and $h_{i, N}^{(\mathrm{iv})}$ is continuous. Hence, since $h_{i, N}$ is $b$-periodic, $h_{i, N} \in H_{\#}^{4}(0, b)$ and $h_{i, N}^{(\mathrm{iv})}$ is $b$-periodic too.

Differentiating (3.22), we obtain

$$
f_{i, N}^{\prime \prime}(x)=a_{i, N} \int_{0}^{x} J_{i-1, N} d \zeta+2 c_{i, N}
$$

By (3.21), the $b$-periodicity of $h_{i, N}$, its derivatives up to order $4, Q_{i, N}$, and $H_{i, N}$, we conclude that $f_{i, N}^{\prime \prime}$ is $b$-periodic and so $a_{i, N}=0$.

Differentiating twice $(3.21)$, we obtain

$$
\varepsilon\left(\frac{h_{i, N}^{\prime \prime}}{J_{i, N}^{5}}\right)^{\prime \prime}+\left(\frac{5 \varepsilon}{2} \frac{\left(h_{i, N}^{\prime \prime}\right)^{2}}{J_{i, N}^{7}} h_{i, N}^{\prime}+\psi_{x}\left(-h_{i, N}^{\prime}, 1\right)\right)^{\prime}+Q_{i, N}-H_{i, N}=2 c_{i, N}
$$

Integrating both sides of the above equality in $(0, b)$ and using periodicity, we deduce

$$
2 b c_{i, N}=\int_{0}^{b}\left(Q_{i, N}-H_{i, N}\right) d x
$$


From (3.24), using Theorem 3.4 and Young inequality to estimate $h_{i, N}^{\prime}, J_{i, N}$, and $Q_{i, N}$, we have

$$
\begin{aligned}
\int_{0}^{b}\left|h_{i, N}^{(\mathrm{iv})}\right|^{2} d x & \leq C \int_{0}^{b}\left(1+\left|h_{i, N}^{\prime \prime}\right|^{6}+\left|h_{i, N}^{\prime \prime}\right|^{2}\left|h_{i, N}^{\prime \prime \prime}\right|^{2}+H_{i, N}^{2}\right) d x \\
& \leq C \int_{0}^{b}\left|h_{i, N}^{\prime \prime \prime}\right|^{3} d x+C \int_{0}^{b}\left|h_{i, N}^{\prime \prime}\right|^{6} d x+C \int_{0}^{b}\left(1+H_{i, N}^{2}\right) d x .
\end{aligned}
$$

Using Theorem 6.4 to estimate $\left\|h_{i, N}^{\prime \prime}\right\|_{L^{6}}$ and $\left\|h_{i, N}^{\prime \prime \prime}\right\|_{L^{3}}$, we obtain from (3.25)

$$
\begin{aligned}
\left\|h_{i, N}^{(\mathrm{iv})}\right\|_{L^{2}}^{2} & \leq C\left\|h_{i, N}^{\prime \prime}\right\|_{L^{2}}^{\frac{5}{4}}\left\|h_{i, N}^{(\mathrm{iv})}\right\|_{L^{2}}^{\frac{7}{4}}+C\left\|h_{i, N}^{\prime \prime}\right\|_{L^{2}}^{5}\left\|h_{i, N}^{(\mathrm{iv})}\right\|_{L^{2}}+C \int_{0}^{b}\left(1+H_{i, N}^{2}\right) d x \\
& \leq \eta\left\|h_{i, N}^{(\mathrm{iv})}\right\|_{L^{2}}^{2}+C_{\eta} \int_{0}^{b}\left(1+H_{i, N}^{2}\right) d x
\end{aligned}
$$

where in the last inequality we used Theorem 3.4-(ii). Choosing $\eta$ sufficiently small and summing over all $i$ such that $i \frac{T}{N} \leq T_{0}$, and recalling (3.5), we finally conclude that

$$
\int_{0}^{T_{0}} \int_{0}^{b}\left|\frac{\partial^{4} h_{N}}{\partial x^{4}}(x, t)\right|^{2} d x d t \leq C \int_{0}^{T_{0}} \int_{0}^{b}\left(1+H_{N}^{2}\right) d x d t \leq C
$$

thus proving (3.18).

Remark 3.6. Denote by $\tilde{h}_{N}$ the piecewise interpolation function

$$
\tilde{h}_{N}(x, t):=\sum_{i=1}^{N} \chi_{\left[\frac{i-1}{N} T, \frac{i}{N} T\right)}(t) h_{i, N}(x) .
$$

The argument used in the proof of Theorem 3.5 allows us to write in place of (3.26) the following inequality

$$
\int_{0}^{T_{0}} \int_{0}^{b}\left|\frac{\partial^{4} \tilde{h}_{N}}{\partial x^{4}}(x, t)\right|^{2} d x d t \leq C .
$$

Note also that property (i) in Theorem 3.4 implies that $\tilde{h}_{N} \rightarrow h$ in $L^{\infty}\left(0, T_{0} ; C^{1, \alpha}([0, b])\right)$ for all $\alpha \in\left(0, \frac{1}{2}\right)$.

As a consequence of estimate (3.18) we get that the discrete time evolutions $h_{N}$ converge to $h$ in a much stronger sense.

Corollary 3.7. Let $T_{0}>0$ be as in Theorem 3.4. Then

$$
h_{N}, \tilde{h}_{N} \rightarrow h \quad \text { in } L^{\frac{12}{5}}\left(0, T_{0} ; C_{\#}^{2,1}([0, b])\right) \cap L^{\frac{24}{5}}\left(0, T_{0} ; C_{\#}^{1,1}([0, b])\right) .
$$

Proof. Fix $N>M$. By applying Theorem 6.4, we get that

$$
\begin{aligned}
& \left\|\frac{\partial^{3} h_{N}}{\partial x^{3}}(\cdot, t)-\frac{\partial^{3} h_{M}}{\partial x^{3}}(\cdot, t)\right\|_{L^{\infty}(0, b)} \\
& \quad \leq C\left(\int_{0}^{b}\left|\frac{\partial^{4} h_{N}}{\partial x^{4}}(x, t)-\frac{\partial^{4} h_{M}}{\partial x^{4}}(x, t)\right|^{2} d x\right)^{\frac{5}{12}}\left(\int_{0}^{b}\left|\frac{\partial h_{N}}{\partial x}(x, t)-\frac{\partial h_{M}}{\partial x}(x, t)\right|^{2} d x\right)^{\frac{1}{12}} .
\end{aligned}
$$

Raising both sides to the power $\frac{12}{5}$, integrating with respect to time, and recalling (3.18) we have that

$$
\int_{0}^{T_{0}}\left\|\frac{\partial^{3} h_{N}}{\partial x^{3}}(\cdot, t)-\frac{\partial^{3} h_{M}}{\partial x^{3}}(\cdot, t)\right\|_{L^{\infty}(0, b)}^{\frac{12}{5}} d t \leq C \int_{0}^{T_{0}}\left\|\frac{\partial h_{N}}{\partial x}(\cdot, t)-\frac{\partial h_{M}}{\partial x}(\cdot, t)\right\|_{L^{\infty}(0, b)}^{\frac{2}{5}} d t
$$


The conclusion then follows from property (i) in Theorem 3.4. This proves the convergence of $h_{N}$ to $h$ in $L^{\frac{12}{5}}\left(0, T_{0} ; C_{\#}^{2,1}([0, b])\right)$. The convergence of $h_{N}$ to $h$ in $L^{\frac{24}{5}}\left(0, T_{0} ; C_{\#}^{1,1}([0, b])\right)$ is obtained similarly by observing that from Theorem 6.4

$\left\|\frac{\partial^{2} h_{N}}{\partial x^{2}}(\cdot, t)-\frac{\partial^{2} h_{M}}{\partial x^{2}}(\cdot, t)\right\|_{L^{\infty}(0, b)} \leq C\left\|\frac{\partial^{3} h_{N}}{\partial x^{3}}(\cdot, t)-\frac{\partial^{3} h_{M}}{\partial x^{3}}(\cdot, t)\right\|_{L^{\infty}(0, b)}^{\frac{1}{2}}\left\|\frac{\partial h_{N}}{\partial x}(\cdot, t)-\frac{\partial h_{M}}{\partial x}(\cdot, t)\right\|_{L^{\infty}(0, b)}^{\frac{1}{2}}$

for almost every $t$. Finally, the corresponding convergence for $\tilde{h}_{N}$ are proved analogously in view of Remark 3.6.

The next theorem establishes the existence of a weak solution to $(3.1)$ for all $h_{0} \in H_{\#}^{2}(0, b)$ strictly positive.

Theorem 3.8. Let $T_{0}$ be as in Theorem 3.4. Then equation (3.1) admits a weak solution in $\left[0, T_{0}\right]$ in the sense of Definition 3.1 with initial datum $h_{0}$. Moreover, if $\psi \in C^{3}\left(\mathbb{R}^{2} \backslash\{0\}\right)$ then $h(\cdot, t) \in H_{\#}^{5}(0, b)$ for almost every $t \in\left[0, T_{0}\right]$.

Proof. Define

$$
\tilde{H}_{N}(x, t):=H_{i, N}(x), \quad \tilde{Q}_{N}(x, t):=Q_{i, N}(x), \quad \tilde{J}_{N}(x, t):=J_{i, N}(x) \quad \text { if }(i-1) \frac{T}{N} \leq t<i \frac{T}{N},
$$

where $H_{i, N}$ and $Q_{i, N}$ have been introduced in (3.20). Fix $t \in\left(0, T_{0}\right)$ and a sequence $\left(j_{n}, N_{n}\right)$ such that $\frac{j_{n}}{N_{n}} T \rightarrow t$. Fix also $\chi \in C_{c}^{\infty}(0, b)$ and $\varphi_{i, N_{n}}:=\left(\frac{\chi^{\prime}}{J_{i-1, N_{n}}}\right)^{\prime}$. Define also $\tilde{\varphi}_{n}(x, t):=\varphi_{i, N_{n}}(x)$ if $(i-1) \frac{T}{N} \leq t<i \frac{T}{N}$. Inserting $\varphi_{i, N_{n}}$ in (3.19) and summing up the resulting equations from $i=1$ to $i=j_{n}$, we get

$$
\begin{array}{r}
\int_{0}^{t} \int_{0}^{b}\left[\varepsilon \frac{\left(\tilde{h}_{N_{n}}\right)_{x x}}{\tilde{J}_{N_{n}}^{5}}\left(\tilde{\varphi}_{n}\right)_{x x}-\frac{5 \varepsilon}{2} \frac{\left(\tilde{h}_{N_{n}}\right)_{x x}^{2}}{\tilde{J}_{N_{n}}^{7}}\left(\tilde{h}_{N_{n}}\right)_{x}\left(\tilde{\varphi}_{n}\right)_{x}-\psi_{x}\left(-\left(\tilde{h}_{N_{n}}\right)_{x}, 1\right)\left(\tilde{\varphi}_{n}\right)_{x}\right] d x d \tau \\
+\int_{0}^{t} \int_{0}^{b}\left(\tilde{Q}_{N_{n}}-\tilde{H}_{N_{n}}\right) \tilde{\varphi}_{n} d x d \tau=0
\end{array}
$$

Integrating by parts the first term in the integral, we obtain

$$
\begin{array}{r}
\int_{0}^{t} \int_{0}^{b}\left[-\varepsilon\left(\frac{\left(\tilde{h}_{N_{n}}\right)_{x x}}{\tilde{J}_{N_{n}}^{5}}\right)_{x}\left(\tilde{\varphi}_{n}\right)_{x}-\frac{5 \varepsilon}{2} \frac{\left(\tilde{h}_{N_{n}}\right)_{x x}^{2}}{\tilde{J}_{N_{n}}^{7}}\left(\tilde{h}_{N_{n}}\right)_{x}\left(\tilde{\varphi}_{n}\right)_{x}-\psi_{x}\left(-\left(\tilde{h}_{N_{n}}\right)_{x}, 1\right)\left(\tilde{\varphi}_{n}\right)_{x}\right] d x d \tau \\
+\int_{0}^{t} \int_{0}^{b}\left(\tilde{Q}_{N_{n}}-\tilde{H}_{N_{n}}\right) \tilde{\varphi}_{n} d x d \tau=0 .
\end{array}
$$

To pass to the limit in this equation we recall the following facts:

(i) by Corollary 3.7 we have that $\left(\frac{\left(\tilde{h}_{N_{n}}\right)_{x x}}{\tilde{J}_{N_{n}}^{5}}\right)_{x} \rightarrow\left(\frac{h_{x x}}{J^{5}}\right)_{x}$ in $L^{\frac{12}{5}}\left(0, T_{0} ; L^{\infty}(0, b)\right)$;

(ii) by $(3.8) \tilde{H}_{N}$ converges weakly in $L^{2}\left(0, T_{0} ; H_{\#}^{1}(0, b)\right)$ to the unique function $H$ such that $H(0, t)=H(b, t)=0$ and

$$
\left(\frac{H_{x}}{J}\right)_{x}=\frac{\partial h}{\partial t} \text { in } H_{\#}^{-1}(0, b)
$$

for almost every $t$. Indeed, from (3.20) we have that

$$
\left(\frac{\left(H_{i, N}\right)_{x}}{J_{i-1, N}}\right)_{x}=\frac{\partial h_{N}}{\partial t} \quad \text { for } t \in\left[\frac{i-1}{N} T, \frac{i}{N} T\right)
$$

and (3.27) easily follows by (3.8) and property (i) in Theorem 3.4.

(iii) by property (iv) of Theorem 3.4 we have that $\tilde{Q}_{N} \rightarrow Q(E(u(\cdot, h(\cdot, \tau))))=: Q(\cdot, \tau)$ in $L^{\infty}\left(0, T_{0} ; C^{1, \alpha}([0, b])\right)$ for all $\alpha \in\left(0, \frac{1}{2}\right)$;

(iv) by Corollary $3.7\left(\tilde{\varphi}_{n}\right)_{x} \rightarrow \varphi_{x}$ in $L^{\frac{12}{5}}\left(0, T_{0} ; L^{\infty}(0, b)\right)$, where $\varphi(x, t):=\left(\frac{\chi^{\prime}(x)}{J(x, t)}\right)_{x}$. 
Therefore, passing to the limit in $n$, we get

$$
\int_{0}^{t} \int_{0}^{b}\left[-\varepsilon\left(\frac{h_{x x}}{J^{5}}\right)_{x}-\frac{5 \varepsilon}{2} \frac{\left(h_{x x}\right)^{2}}{J^{7}} h_{x}-\psi_{x}\left(-h_{x}, 1\right)\right] \varphi_{x} d x d \tau+\int_{0}^{t} \int_{0}^{b}(Q-H) \varphi d x d \tau=0 .
$$

From (3.28) it follows that for every $t \in\left(0, T_{0}\right) \backslash N_{\chi}$, with $\mathcal{L}^{1}\left(N_{\chi}\right)=0$,

$$
\int_{0}^{b}\left[-\varepsilon\left(\frac{h_{x x}}{J^{5}}\right)_{x}-\frac{5 \varepsilon}{2} \frac{\left(h_{x x}\right)^{2}}{J^{7}} h_{x}-\psi_{x}\left(-h_{x}, 1\right)\right] \varphi_{x} d x+\int_{0}^{b}(Q-H) \varphi d x=0 .
$$

Letting $\chi$ vary in a countable dense subset of $C_{c}^{\infty}(0, b)$ we conclude that for almost every $t \in\left[0, T_{0}\right]$ equation (3.29) holds for all $\chi \in C_{c}^{\infty}(0, b)$ and thus

$$
\int_{0}^{b}\left[-\varepsilon\left(\frac{h_{x x}}{J^{5}}\right)_{x}-\frac{5 \varepsilon}{2} \frac{\left(h_{x x}\right)^{2}}{J^{7}} h_{x}-\psi_{x}\left(-h_{x}, 1\right)\right] \eta^{\prime} d x+\int_{0}^{b}(Q-H) \eta d x=0
$$

for all $\eta \in C_{c}^{2}([0, b])$ such that $\int_{0}^{b} \eta d x=0$ and $\int_{\Gamma_{h}} \int_{0}^{x} \eta d \zeta d \mathcal{H}^{1}=0$.

We claim that from $(3.30)$ it follows that $h(\cdot, t) \in C_{\#}^{4}([0, b])$ and

$$
\varepsilon\left(\frac{h_{x x}}{J^{5}}\right)_{x x}+\frac{5 \varepsilon}{2}\left(\frac{h_{x x}^{2}}{J^{7}} h_{x}\right)_{x}+\left(\psi_{x}\left(-h_{x}, 1\right)\right)_{x}+Q-H=\alpha \sigma+\beta
$$

for suitable time-dependent functions $\alpha=\alpha(t)$ and $\beta=\beta(t)$. Here, $\sigma(x, t):=\int_{0}^{x} J(x, \tau) d \tau$. To prove the claim note that since by Theorem $3.5 h \in L^{2}\left(0, T_{0} ; H_{\#}^{4}(0, b)\right)$, (3.30) can be written as

$$
\int_{0}^{b} f \eta d x=0 \text { for all } \eta \text { as above, }
$$

where

$$
f(x):=\varepsilon\left(\frac{h_{x x}}{J^{5}}\right)_{x x}+\frac{5 \varepsilon}{2}\left(\frac{h_{x x}^{2}}{J^{7}} h_{x}\right)_{x}+\left(\psi_{x}\left(-h_{x}, 1\right)\right)_{x}+Q-H .
$$

By Lemma $6.8,(3.31)$ follows. Note that from $(3.31)$, recalling that $\psi \in C^{2}\left(\mathbb{R}^{2} \backslash\{0\}\right)$, we obtain that $h(\cdot, t) \in C^{4}(0, b)$. Now using equation (3.23) in any interval of the form $(a, a+b)$ and arguing as before, we deduce that $h(\cdot, t) \in C^{4}(\mathbb{R})$. In particular, $h(\cdot, t) \in C_{\#}^{4}([0, b])$. In turn, the left-hand side of (3.31) is $b$-periodic, and since $H$ is also $b$-periodic we conclude that $\alpha$ must be zero. Note that since $h(\cdot, t) \in C_{\#}^{4}([0, b])$, by elliptic regularity we have that $u(\cdot, t) \in C^{3}\left(\bar{\Omega}_{h(\cdot, t)} ; \mathbb{R}^{2}\right)$. Therefore, since $H(\cdot, t) \in H_{\#}^{1}(0, b)$, from equation $(3.31)$ we deduce that $h(\cdot, t) \in H_{\#}^{5}(0, b)$ if $\psi \in C^{3}\left(\mathbb{R}^{2} \backslash\{0\}\right)$.

Integrating (3.31) with respect to $x$ and recalling that $\alpha=0$, we get that

$$
\beta(t)=\frac{1}{b} \int_{0}^{b}(Q(x, t)-H(x, t)) d x .
$$

Therefore, since $H \in L^{2}\left(0, T_{0} ; H_{\#}^{1}(0, b)\right)$ we deduce that

$$
\varepsilon\left(\frac{h_{x x}}{J^{5}}\right)_{x x}+\frac{5 \varepsilon}{2}\left(\frac{h_{x x}^{2}}{J^{7}} h_{x}\right)_{x}+\left(\psi_{x}\left(-h_{x}, 1\right)\right)_{x}+Q \in L^{2}\left(0, T_{0} ; H_{\#}^{1}(0, b)\right)
$$

thus proving condition (ii) in Definition 3.1, thanks to part (i) of Remark 3.2.

Finally, in view of part (i) of Remark 3.2, condition (iii) in Definition 3.1 follows by combining (3.27) with (3.31), recalling that $\alpha=0$. 


\section{Regularity}

In this section we show that if $\psi$ is of class $C^{4}$ away from the origin and $h_{0} \in H_{\#}^{3}(0, b), h_{0}>0$, then the solution constructed in the previous section satisfies (3.1) in a stronger sense, precisley, $h \in L^{2}\left(0, T_{0} ; H_{\#}^{6}(0, b)\right) \cap H^{1}\left(0, T_{0} ; L^{2}(0, b)\right)$.

Next we need a delicate and crucial elliptic estimate on the the trace of the $E(u)$ on $\Gamma_{h}$, where $u$ is the elastic equilibrium in $\Omega_{h}$.

Theorem 4.1. Let $(h, u) \in X_{e_{0}}$ be such that $h \in H_{\#}^{5}(0, b), h \geq c_{0}>0$, and $u$ is the corresponding elastic equilibrium. Then, there exists a constant $C$ depending only on $\|h\|_{H^{2}(0, b)}, c_{0}$, and $\|E(u)\|_{L^{\infty}\left(\Omega_{h}\right)}$ such that the following estimates hold:

$$
\int_{\Gamma_{h}}|D E(u)|^{2} d \mathcal{H}^{1} \leq C \int_{0}^{b}\left(1+\left|h^{(\mathrm{iv})}\right|^{2}\right) d x
$$

and

$$
\int_{\Gamma_{h}}\left|D^{2} E(u)\right|^{2} d \mathcal{H}^{1}+\int_{\Gamma_{h}}\left|D_{\sigma}(E(u))\right|^{4} d \mathcal{H}^{1} \leq C \int_{0}^{b}\left(1+\left|h^{(\mathrm{v})}\right|^{2}\right) d x
$$

where $D_{\sigma}$ denotes the tangential derivative along $\Gamma_{h}$.

Proof. We split the proof into several steps.

Step 1. (Airy Functions) Since $h \in H_{\#}^{5}(0, b)$, by elliptic regularity we have that $u \in C^{4, \frac{1}{2}}\left(\bar{\Omega}_{h}^{\#}\right)$. We now introduce the Airy function $w$ associated to $u$, which satisfies

$$
\left(\begin{array}{ll}
w_{x x} & w_{x y} \\
w_{x y} & w_{y y}
\end{array}\right)=\left(\begin{array}{cc}
(\mathbb{C} E(u))_{22} & -(\mathbb{C} E(u))_{12} \\
-(\mathbb{C} E(u))_{12} & (\mathbb{C} E(u))_{11}
\end{array}\right) .
$$

It is well known that $w$ is biharmonic (see for instance [20, Ch. 12]). Note that we can choose $w$ to satisfy the additional conditions

$$
w=0 \quad \text { and } \quad \nabla w=0 \quad \text { on } \Gamma_{h}^{\#} .
$$

Indeed, since $\mathbb{C} E(u)[\nu]=0$ on $\Gamma_{h}^{\#}$, it follows from (4.3) that $D_{\sigma}(\nabla w)=0$. Hence, by a subtracting a suitable affine function, if necessary, we may impose that (4.4) holds. Moreover, from the regularity of $u$ we have that $w \in C^{5, \frac{1}{2}}\left(\Omega_{h}^{\#}\right)$.

Step 2. (Straightening the boundary) We denote by $Q_{h}^{\#}$ the image of $\Omega_{h}^{\#}$ under the diffeomorphism $\Phi_{h}(x, y):=(x, y-h(x))$. Note that $\Phi_{h}\left(\Gamma_{h}^{\#}\right)=\{y=0\}$. Set $v(x, y):=w(x, y+h(x))$ for all $(x, y) \in Q_{h}^{\#}$. Note that, since

$$
v_{x}=w_{x}+h^{\prime} w_{y}, \quad v_{y}=w_{y},
$$

and

$$
v_{x x}=w_{x x}+2 h^{\prime} w_{x y}+h^{\prime 2} w_{y y}+h^{\prime \prime} w_{y}, \quad v_{x y}=w_{x y}+h^{\prime} w_{y y}, \quad v_{y y}=w_{y y},
$$

from the assumptions on $h$ and $u$ we conclude that

$$
|D v| \leq C \quad \text { and } \quad\left|D^{2} v\right| \leq C\left(1+\left|h^{\prime \prime}\right|\right)
$$

for some constant $C>0$ depending only on $\left\|h^{\prime}\right\|_{L^{\infty}}$ and $\|E(u)\|_{L^{\infty}}$. Since $w(x, y)=v(x, y-h(x))$ and $\Delta^{2} w=0$, a lengthy but straightforward calculation shows that $v$ satisfies the 4 -th order elliptic equation $L(v)=f$ in $Q_{h}^{\#}$, where

$$
f:=6 h^{\prime \prime} v_{x x y}-12 h^{\prime} h^{\prime \prime} v_{x y y}+\left(2+6 h^{2}\right) h^{\prime \prime} v_{y y y}+4 h^{\prime \prime \prime} v_{x y}-\left(3 h^{\prime \prime 2}+4 h^{\prime} h^{\prime \prime \prime}\right) v_{y y}+h^{(\mathrm{iv})} v_{y},
$$

and

$$
L(v):=v_{x x x x}-4 h^{\prime} v_{x x x y}+\left(2+6 h^{\prime 2}\right) v_{x x y y}-\left(4 h^{\prime}+4 h^{\prime 3}\right) v_{x y y y}+\left(1+h^{\prime 2}\right)^{2} v_{y y y y} .
$$


Moreover, $v$ satisfies the boundary conditions (see (4.4))

$$
v=0 \quad \text { and } \quad \nabla v=0 \quad \text { on }\{y=0\} .
$$

Set $S:=[0, b] \times\{0\}$, fix two bounded open sets $U^{\prime}$ and $V^{\prime}$ such that $S \subset U^{\prime} \subset \subset V^{\prime}$, with $\bar{V}^{\prime} \cap \Phi_{h}(\{y=0\})=\emptyset$, and denote $U:=U^{\prime} \cap Q_{h}^{\#}$ and $V:=V^{\prime} \cap Q_{h}^{\#}$. From well-known elliptic estimates (see [2]) we have that

$$
\int_{U}\left|D^{4} v\right|^{2} d z \leq C \int_{V}\left(|v|^{2}+|f|^{2}\right) d z
$$

Step 3. (Estimate of $D^{4} v$ ) From (4.8) and (4.9), recalling (4.6) and using Young's inequality, we get

$$
\begin{aligned}
\int_{U}\left|D^{4} v\right|^{2} d z & \leq C \int_{V}\left[1+\left|h^{(\mathrm{iv})}\right|^{2}|D v|^{2}+\left(h^{\prime \prime 4}+h^{\prime \prime \prime 2}\right)\left|D^{2} v\right|^{2}+h^{\prime \prime 2}\left|D^{3} v\right|^{2}\right] d z \\
& \leq C_{\eta} \int_{0}^{b}\left(1+h^{\prime \prime 6}+h^{\prime \prime \prime 2}+h^{\prime \prime 2} h^{\prime \prime \prime 2}+\left|h^{(\mathrm{iv})}\right|^{2}\right) d x+\eta \int_{V}\left|D^{3} v\right|^{3} d z \\
& \leq C_{\eta} \int_{0}^{b}\left(1+h^{\prime \prime 6}+h^{\prime \prime \prime 3}+\left|h^{(\mathrm{iv})}\right|^{2}\right) d x+\eta \int_{V}\left|D^{3} v\right|^{3} d z
\end{aligned}
$$

We now use Theorem 6.4 to estimate

$$
\int_{0}^{b}\left(h^{\prime \prime 6}+h^{\prime \prime \prime 3}\right) d x \leq C\left(\left\|h^{(\mathrm{iv})}\right\|_{L^{2}}\left\|h^{\prime \prime}\right\|_{L^{2}}^{5}+\left\|h^{(\mathrm{iv})}\right\|_{L^{2}}^{\frac{7}{4}}\left\|h^{\prime \prime}\right\|_{L^{2}}^{\frac{5}{4}}\right) \leq C \int_{0}^{b}\left(1+\left|h^{(\mathrm{iv})}\right|^{2}\right) d x
$$

for a suitable constant $C>0$ depending only on $\left\|h^{\prime \prime}\right\|_{L^{2}}$. Using Theorem 6.4 and (4.6), we have

$$
\begin{aligned}
\int_{V}\left|D^{3} v\right|^{3} d z & \leq C \int_{V}\left|D^{4} v\right|^{2} d z\left(\int_{V}\left|D^{2} v\right|^{2} d z\right)^{\frac{1}{2}}+\left(\int_{V}\left|D^{2} v\right|^{2} d z\right)^{\frac{3}{2}} \\
& \leq C \int_{V}\left|D^{4} v\right|^{2} d z\left(\int_{0}^{b}\left(1+h^{\prime \prime 2}\right) d x\right)^{\frac{1}{2}}+C\left(\int_{0}^{b}\left(1+h^{\prime \prime 2}\right) d x\right)^{\frac{3}{2}} \leq C \int_{V}\left(1+\left|D^{4} v\right|^{2}\right) d z
\end{aligned}
$$

for some constant $C>0$ depending only $\|E(u)\|_{L^{\infty}}$ and $\left\|h^{\prime \prime}\right\|_{L^{2}}$. Inserting this estimate in (4.10), recalling (4.11), and taking $\eta$ sufficiently small, we conclude that

$$
\int_{U}\left|D^{4} v\right|^{2} d z \leq C \int_{0}^{b}\left(1+\left|h^{(\mathrm{iv})}\right|^{2}\right) d x+C \int_{V \backslash U}\left|D^{4} v\right|^{2} d z
$$

Step 4. (Estimate of $D^{5} v$ ) Differentiating equation (4.7) with respect to $x$, since $v_{x}=0$ and $\nabla v_{x}=0$ on $\{y=0\}$, as before from elliptic regularity we have

$$
\int_{U}\left|D^{4} v_{x}\right|^{2} d z \leq C \int_{V}\left(1+\left|v_{x}\right|^{2}+\left|f_{x}\right|^{2}+h^{\prime \prime 2}\left|D^{4} v\right|^{2}\right) d z .
$$

From (4.7), recalling (4.6) and using Young's inequality, we get

$$
\begin{aligned}
\int_{V}\left(\left|f_{x}\right|^{2}+h^{\prime \prime 2}\left|D^{4} v\right|^{2}\right) d z \leq C \int_{V} & {\left[\left|h^{(\mathrm{v})}\right|^{2}|D v|^{2}+\left(h^{\prime \prime 2} h^{\prime \prime \prime 2}+\left|h^{(\mathrm{iv})}\right|^{2}\right)\left|D^{2} v\right|^{2}\right] d z } \\
& +C \int_{V}\left[\left(1+h^{\prime \prime 4}+h^{\prime \prime \prime 2}\right)\left|D^{3} v\right|^{2}+h^{\prime \prime 2}\left|D^{4} v\right|^{2}\right] d z \\
\leq \eta \int_{V}\left(\left|D^{3} v\right|^{4}+\left|D^{4} v\right|^{\frac{8}{3}}\right) d z & +C_{\eta} \int_{0}^{b}\left(1+h^{\prime \prime 8}+h^{\prime \prime \prime 4}+\left|h^{(\mathrm{iv})}\right|^{\frac{8}{3}}+\left|h^{(\mathrm{v})}\right|^{2}\right) d x
\end{aligned}
$$


Using repeatedly Theorem 6.4 , we get

$$
\begin{aligned}
\int_{0}^{b}\left(h^{\prime \prime 8}+h^{\prime \prime \prime 4}+\left|h^{(\mathrm{iv})}\right|^{\frac{8}{3}}\right) d x & \leq C\left(\left\|h^{(\mathrm{v})}\right\|_{L^{2}}\left\|h^{\prime \prime}\right\|_{L^{2}}^{7}+\left\|h^{(\mathrm{v})}\right\|_{L^{2}}^{\frac{5}{3}}\left\|h^{\prime \prime}\right\|_{L^{2}}^{\frac{7}{3}}+\left\|h^{(\mathrm{v})}\right\|_{L^{2}}^{\frac{17}{9}}\left\|h^{\prime \prime}\right\|_{L^{2}}^{\frac{7}{9}}\right) \\
& \leq C \int_{0}^{b}\left(1+\left|h^{(\mathrm{v})}\right|^{2}\right) d x
\end{aligned}
$$

for some constant $C$ depending only on $\|h\|_{H^{2}}$. Similarly, using Theorem 6.4 and again (4.6),

$$
\begin{aligned}
& \int_{V}\left(\left|D^{3} v\right|^{4}+\left|D^{4} v\right|^{\frac{8}{3}}\right) d z \\
& \leq C\left(\left\|D^{5} v\right\|_{L^{2}(V)}^{2}\left\|D^{2} v\right\|_{L^{2}(V)}^{2}+\left\|D^{2} v\right\|_{L^{2}(V)}^{4}+\left\|D^{5} v\right\|_{L^{2}(V)}^{2}\left\|D^{2} v\right\|_{L^{2}(V)}^{\frac{2}{3}}+\left\|D^{2} v\right\|_{L^{2}(V)}^{\frac{8}{3}}\right) \\
& \leq C\left(1+\left\|D^{5} v\right\|_{L^{2}(V)}^{2}\right) .
\end{aligned}
$$

Inserting the last estimate and (4.15) in (4.14), and recalling (4.13), we have

$$
\int_{U}\left|D^{4} v_{x}\right|^{2} d z \leq C \eta \int_{V}\left|D^{5} v\right|^{2} d z+C_{\eta} \int_{0}^{b}\left(1+\left|h^{(\mathrm{v})}\right|^{2}\right) d x .
$$

Differentiating (4.7) with respect to $y$, we easily get

$$
\left\|v_{\text {yyyyy }}\right\|_{L^{2}(U)}^{2} \leq C \int_{U}\left|D^{4} v_{x}\right|^{2} d z+\int_{U}\left[h^{\prime \prime 2}\left|D^{4} v\right|^{2}+\left(h^{\prime \prime 4}+h^{\prime \prime \prime 2}\right)\left|D^{3} v\right|^{2}+\left|h^{(\mathrm{iv})}\right|^{2}\left|D^{2} v\right|^{2}\right] d z .
$$

Estimating the second integral in the previous formula as we did for the right-hand side of (4.14) and recalling (4.16), we have

$$
\int_{U}\left|D^{5} v\right|^{2} d z \leq C \eta \int_{V}\left|D^{5} v\right|^{2} d z+C_{\eta} \int_{0}^{b}\left(1+\left|h^{(\mathrm{v})}\right|^{2}\right) d x .
$$

Choosing $\eta$ small enough, we conclude that

$$
\int_{U}\left|D^{5} v\right|^{2} d z \leq C \int_{0}^{b}\left(1+\left|h^{(\mathrm{v})}\right|^{2}\right) d x+C \int_{V \backslash U}\left|D^{5} v\right|^{2} d z .
$$

Step 5. (Proof of (4.1)) Let $W^{\prime}$ be an open set such that $\Phi_{h}^{-1}(V \backslash U) \subset \subset W^{\prime}$ and set $W:=W^{\prime} \cap \Omega_{h}^{\#}$. We first observe that since $u \in e_{0}(\cdot, 0)+L D_{\#}\left(\Omega_{h} ; \mathbb{R}^{2}\right)$ solves the linear system

$$
\left\{\begin{array}{l}
\int_{\Omega_{h}} \mathbb{C} E(u): E(\varphi)=0 \quad \text { for all } \varphi \in L D_{\#}\left(\Omega_{h} ; \mathbb{R}^{2}\right) \text { s.t. } \varphi(x, 0)=0 \\
u(x, 0)=e_{0}(x, 0)
\end{array}\right.
$$

then standard elliptic regularity implies that for all $n \in \mathbb{N}$

$$
\int_{\Phi_{h}^{-1}(V \backslash U)}\left|D^{n} u\right|^{2} d z \leq C \int_{W}\left(1+|u|^{2}\right) d z \leq C
$$

for some positive constant $C$ depending only on $n$ and $e_{0}$. Therefore, if $n \geq 2$ from (4.3) it follows that

$$
\int_{\Phi_{h}^{-1}(V \backslash U)}\left|D^{n} w\right|^{2} d z \leq C \int_{\Phi_{h}^{-1}(V \backslash U)}\left|D^{n-1} u\right|^{2} d z \leq C
$$

for some constant depending only on $n$ and $e_{0}$. Observe that similarly to (4.5) we have

$$
w_{x x}=v_{x x}-2 h^{\prime} v_{x y}+h^{\prime 2} v_{y y}-h^{\prime \prime} v_{y}, \quad w_{x y}=v_{x y}-h^{\prime} v_{y y}, \quad \text { and } \quad w_{y y}=v_{y y} .
$$


Differentiating these inequalities with respect to $x$ and $y$ and using (4.3) and (4.6), we get

$$
\begin{aligned}
\int_{\Gamma_{h}}|D E(u)|^{2} d \mathcal{H}^{1} & \leq C \int_{\Gamma_{h}}\left|D^{3} w\right|^{2} d \mathcal{H}^{1} \leq C \int_{S}\left(\left|D^{3} v\right|^{2}+\left|D^{2} v\right|^{2} h^{\prime \prime 2}+|D v|^{2} h^{\prime \prime \prime 2}\right) d x \\
& \leq C \int_{U}\left(\left|D^{3} v\right|^{2}+\left|D^{4} v\right|^{2}\right) d z+C \int_{0}^{b}\left(1+h^{\prime \prime 4}+h^{\prime \prime \prime 2}\right) d x \\
& \leq C \int_{U}\left(\left|D^{2} v\right|^{2}+\left|D^{4} v\right|^{2}\right) d z+C \int_{0}^{b}\left(1+h^{\prime \prime 4}+h^{\prime \prime \prime 2}\right) d x \\
& \leq C \int_{U}\left|D^{4} v\right|^{2} d z+C \int_{0}^{b}\left(1+h^{\prime \prime 4}+h^{\prime \prime \prime 2}\right) d x
\end{aligned}
$$

where we have used the trace theorem to control $\left\|D^{3} v\right\|_{L^{2}(S)}$ with $\left\|D^{3} v\right\|_{H^{1}(U)}$. Estimating the last integral as we did in Step 3 and recalling (4.12), we obtain

$$
\int_{\Gamma_{h}}|D E(u)|^{2} d \mathcal{H}^{1} \leq C \int_{0}^{b}\left(1+\left|h^{(\mathrm{iv})}\right|^{2}\right) d x+C \int_{V \backslash U}\left|D^{4} v\right|^{2} d z .
$$

Concerning the last integral, we have arguing as in (4.10)

$$
\begin{aligned}
\int_{V \backslash U}\left|D^{4} v\right|^{2} d z & \leq C \int_{\Phi_{h}^{-1}(V \backslash U)}\left[1+\left|h^{(\mathrm{iv})}\right|^{2}|D w|^{2}+\left(h^{\prime \prime 4}+h^{\prime \prime \prime 2}\right)\left|D^{2} w\right|^{2}+h^{\prime \prime 2}\left|D^{3} w\right|^{2}\right] d z \\
& \leq C_{\eta} \int_{0}^{b}\left(1+h^{\prime \prime 6}+h^{\prime \prime \prime 3}+\left|h^{(\mathrm{iv})}\right|^{2}\right) d x+\eta \int_{\Phi_{h}^{-1}(V \backslash U)}\left|D^{3} w\right|^{3} d x \\
& \leq C_{\eta} \int_{0}^{b}\left(1+h^{\prime \prime 6}+h^{\prime \prime \prime 3}+\left|h^{(\mathrm{iv})}\right|^{2}\right) d x+C
\end{aligned}
$$

where we used (4.18). Controlling the last integral by $\int_{0}^{b}\left(1+\left|h^{(\mathrm{iv})}\right|^{2}\right) d x$ as we did in Step 3 (see (4.11)), and inserting the resulting estimate in (4.20), we conclude the proof of (4.1).

Step 6. (Proof of (4.2)) Differentiating (4.19) twice and using (4.3) and (4.6), we have

$$
\begin{aligned}
\int_{\Gamma_{h}}\left|D^{2} E(u)\right|^{2} d \mathcal{H}^{1} & \leq C \int_{\Gamma_{h}}\left|D^{4} w\right|^{2} d \mathcal{H}^{1} \\
& \leq C \int_{S}\left[\left|D^{4} v\right|^{2}+h^{\prime \prime 2}\left|D^{3} v\right|^{2}+\left(h^{\prime \prime 4}+h^{\prime \prime \prime 2}\right)\left|D^{2} v\right|^{2}+\left|h^{(\mathrm{iv})}\right|^{2}|D v|^{2}\right] d x \\
& \leq C \int_{S}\left(\left|D^{3} v\right|^{\frac{8}{3}}+\left|D^{4} v\right|^{2}\right) d x+C \int_{0}^{b}\left(1+h^{\prime \prime 8}+h^{\prime \prime \prime}+\left|h^{(\mathrm{iv})}\right|^{2}\right) d x \\
& \leq C \int_{S}\left(\left|D^{3} v\right|^{\frac{8}{3}}+\left|D^{4} v\right|^{2}\right) d x+C \int_{0}^{b}\left(1+\left|h^{(\mathrm{v})}\right|^{2}\right) d x
\end{aligned}
$$

where the last inequality follows from (4.15). We now estimate, using Theorem 6.9 and Theorem 6.4

$$
\begin{aligned}
\int_{S}\left(\left|D^{3} v\right|^{\frac{8}{3}}+\left|D^{4} v\right|^{2}\right) d x \leq & C\left(\left\|D^{3} v\right\|_{L^{\frac{16}{11}}(U)}^{\frac{8}{3}}+\left\|D^{4} v\right\|_{L^{\frac{16}{11}}(U)}^{\frac{8}{3}}+\left\|D^{4} v\right\|_{L^{2}(U)}^{2}+\left\|D^{5} v\right\|_{L^{2}(U)}^{2}\right) \\
\leq & C\left(\left\|D^{5} v\right\|_{L^{2}(U)}^{\frac{5}{9}}\left\|D^{2} v\right\|_{L^{2}(U)}^{\frac{19}{9}}+\left\|D^{5} v\right\|_{L^{2}(U)}^{\frac{13}{9}}\left\|D^{2} v\right\|_{L^{2}(U)}^{\frac{11}{9}}\right. \\
& \left.+\left\|D^{2} v\right\|_{L^{2}(U)}^{\frac{8}{3}}+\left\|D^{4} v\right\|_{L^{2}(U)}^{2}+\left\|D^{5} v\right\|_{L^{2}(U)}^{2}\right) \\
\leq & C \int_{U}\left(1+\left|D^{4} v\right|^{2}+\left|D^{5} v\right|^{2}\right) d z \leq C \int_{U}\left(1+\left|D^{5} v\right|^{2}\right) d z
\end{aligned}
$$

From this inequality, (4.17), and (4.21), we have

$$
\int_{\Gamma_{h}}\left|D^{2} E(u)\right|^{2} d \mathcal{H}^{1} \leq C \int_{0}^{b}\left(1+\left|h^{(\mathrm{v})}\right|^{2}\right) d x+C \int_{V \backslash U}\left|D^{5} v\right|^{2} d z .
$$


To estimate the tangential derivative of $E(u)$, we observe that by Theorem 6.4

$$
\left\|D_{\sigma} E(u)\right\|_{L^{4}\left(\Gamma_{h}\right)} \leq C\left\|D_{\sigma \sigma}^{2} E(u)\right\|_{L^{4}\left(\Gamma_{h}\right)}^{\frac{1}{2}}\|E(u)\|_{L^{4}\left(\Gamma_{h}\right)}^{\frac{1}{2}} \leq C\left\|D_{\sigma \sigma}^{2} E(u)\right\|_{L^{4}\left(\Gamma_{h}\right)}^{\frac{1}{2}} .
$$

Hence, differentiating twice the identities in (4.19), recalling (4.6) $)_{2}$, and using Young's inequality, we have after some straightforward calculations,

$$
\begin{gathered}
\int_{\Gamma_{h}}\left|D_{\sigma} E(u)\right|^{4} d \mathcal{H}^{1} \leq C\left(\int_{S}\left[\left|D^{4} v\right|^{4}+h^{\prime \prime 4}\left|D^{3} v\right|^{4}+\left(h^{\prime \prime 8}+h^{\prime \prime \prime 4}\right)\left|D^{2} v\right|^{4}+\left|h^{(\mathrm{iv})}\right|^{4}|D v|^{4}\right] d x\right)^{\frac{1}{2}} \\
\leq C\left(\int_{S}\left(\left|D^{3} v\right|^{\frac{24}{5}}+\left|D^{4} v\right|^{4}\right) d x\right)^{\frac{1}{2}}+C\left(\int_{0}^{b}\left(1+h^{\prime \prime 24}+\left|h^{\prime \prime \prime}\right|^{\frac{24}{5}}+\left|h^{(\mathrm{iv})}\right|^{4}\right) d x\right)^{\frac{1}{2}}
\end{gathered}
$$

Using Theorem 6.4, we have

$$
\begin{gathered}
\int_{0}^{b}\left(1+h^{\prime \prime 24}+\left|h^{\prime \prime \prime}\right|^{\frac{24}{5}}+\left|h^{(\mathrm{iv})}\right|^{4}\right) d x \leq C\left(\left\|h^{(\mathrm{v})}\right\|_{L^{2}}^{\frac{11}{3}}\left\|h^{\prime \prime}\right\|_{L^{2}}^{\frac{61}{3}}+\left\|h^{(\mathrm{v})}\right\|_{L^{2}}^{\frac{31}{15}}\left\|h^{\prime \prime}\right\|_{L^{2}}^{\frac{41}{15}}+\left\|h^{(\mathrm{v})}\right\|_{L^{2}}^{3}\left\|h^{\prime \prime}\right\|_{L^{2}}\right) \\
\leq C\left(1+\left\|h^{(\mathrm{v})}\right\|_{L^{2}}^{\frac{11}{3}}\right) \leq C\left(\int_{0}^{b}\left(1+\left|h^{(\mathrm{v})}\right|^{2}\right) d x\right)^{2} .
\end{gathered}
$$

On the other hand, using Theorem 6.9 and (4.6), we can estimate

$$
\begin{aligned}
\int_{S}\left(\left|D^{3} v\right|^{\frac{24}{5}}+\left|D^{4} v\right|^{4}\right) d x & \leq C\left(\left\|D^{3} v\right\|_{W^{1, \frac{48}{29}}(U)}^{\frac{24}{5}}+\left\|D^{4} v\right\|_{W^{1, \frac{8}{5}}(U)}^{4}\right) \\
& \leq C\left(\left\|D^{4} v\right\|_{L^{\frac{48}{29}}(U)}^{\frac{24}{5}}+\left\|D^{3} v\right\|_{L^{\frac{48}{29}}(U)}^{\frac{24}{5}}+\left\|D^{5} v\right\|_{L^{\frac{8}{5}}(U)}^{4}+\left\|D^{4} v\right\|_{L^{\frac{8}{5}}(U)}^{4}\right) \\
& \leq C\left(\left\|D^{4} v\right\|_{L^{\frac{84}{29}}(U)}^{\frac{24}{5}}+\left\|D^{2} v\right\|_{L^{\frac{84}{29}}(U)}^{\frac{24}{4}}+\left\|D^{5} v\right\|_{L^{\frac{8}{5}}(U)}^{4}+\left\|D^{2} v\right\|_{L^{\frac{8}{5}(U)}}^{4}\right) \\
& \leq C\left(1+\left\|D^{4} v\right\|_{L^{\frac{48}{29}(U)}}^{\frac{24}{5}}+\left\|D^{5} v\right\|_{L^{\frac{8}{5}}(U)}^{4}\right) .
\end{aligned}
$$

Combining this estimate with (4.23) and (4.24), using Theorem 6.4, and recalling (4.6), we have

$$
\begin{aligned}
\int_{\Gamma_{h}}\left|D_{\sigma} E(u)\right|^{4} d \mathcal{H}^{1} \leq C\left(\left\|D^{4} v\right\|_{L^{\frac{48}{29}}(U)}^{\frac{12}{5}}+\left\|D^{5} v\right\|_{L^{\frac{8}{5}(U)}}^{2}\right)+C \int_{0}^{b}\left(1+\left|h^{(\mathrm{v})}\right|^{2}\right) d x \\
\leq C\left(\left\|D^{5} v\right\|_{L^{2}(U)}^{\frac{43}{30}}\left\|D^{2} v\right\|_{L^{2}(U)}^{\frac{29}{30}}+\left\|D^{2} v\right\|_{L^{2}(U)}^{\frac{12}{5}}+\left\|D^{5} v\right\|_{L^{\frac{8}{5}(U)}}^{2}\right)+C \int_{0}^{b}\left(1+\left|h^{(\mathrm{v})}\right|^{2}\right) d x \\
\leq C\left\|D^{5} v\right\|_{L^{2}}^{2}+C \int_{0}^{b}\left(1+\left|h^{(\mathrm{v})}\right|^{2}\right) d x
\end{aligned}
$$

The last estimate combined with (4.17) yields

$$
\int_{\Gamma_{h}}\left|D_{\sigma} E(u)\right|^{4} d \mathcal{H}^{1} \leq C \int_{0}^{b}\left(1+\left|h^{(\mathrm{v})}\right|^{2}\right) d x+C \int_{V \backslash U}\left|D^{5} v\right|^{2} d z .
$$

Concerning the last integral, arguing as in (4.14) and (4.15), and using (4.18), we have

$$
\begin{aligned}
& \int_{V \backslash U}\left|D^{5} v\right|^{2} d z \leq C \int_{\Phi_{h}^{-1}(V \backslash U)}\left[\left|h^{(\mathrm{v})}\right|^{2}|D w|^{2}+\left(h^{\prime \prime 2} h^{\prime \prime \prime 2}+\left|h^{(\mathrm{iv})}\right|^{2}\right)\left|D^{2} w\right|^{2}\right] d z \\
& \quad+C \int_{\Phi_{h}^{-1}(V \backslash U)}\left[\left(1+h^{\prime \prime 4}+h^{\prime \prime \prime 2}\right)\left|D^{3} w\right|^{2}+h^{\prime \prime 2}\left|D^{4} w\right|^{2}\right] d z+C \int_{\Phi_{h}^{-1}(V \backslash U)}\left|D^{5} w\right|^{2} d z \\
& \quad \leq C \int_{\Phi_{h}^{-1}(V \backslash U)}\left(\left|D^{3} w\right|^{4}+\left|D^{4} w\right|^{\frac{8}{3}}\right) d z+C \int_{0}^{b}\left(1+h^{\prime \prime 8}+h^{\prime \prime \prime 4}+\left|h^{(\mathrm{iv})}\right|^{2}+\left|h^{(\mathrm{v})}\right|^{2}\right) d x \\
& \leq C \int_{0}^{b}\left(1+\left|h^{(\mathrm{v})}\right|^{2}\right) d x
\end{aligned}
$$


Hence, (4.2) follows by inserting the last estimate in (4.22) and (4.25). This concludes the proof of the theorem.

We now state a regularity result which applies to any weak solution to (3.1).

Theorem 4.2 (Properties of weak solutions). Let $h$ be a weak solution of $(3.1)$ in $\left[0, T_{0}\right]$ in the sense of Definition 3.1. Then, the following properties hold:

(i) $h \in C^{0, \beta}\left(\left[0, T_{0}\right] ; C^{1, \alpha}([0, b])\right)$ for all $\alpha \in\left(0, \frac{1}{2}\right)$ and $\beta \in\left(0, \frac{1-2 \alpha}{16}\right)$;

(ii) $h \in L^{2}\left(0, T_{0} ; H_{\#}^{4}(0, b)\right)$;

(iii) if $\psi \in C^{3}\left(\mathbb{R}^{2} \backslash\{0\}\right)$ then $h \in L^{2}\left(0, T_{0} ; H_{\#}^{5}(0, b)\right)$.

Proof. Property (i) is a consequence of the fact that $H^{1}\left(0, T ; H_{\#}^{-1}(0, b)\right) \cap L^{\infty}\left(0, T ; H_{\#}^{2}(0, b)\right)$ is continuously embedded in $C^{0, \beta}\left([0, T] ; C^{1, \alpha}([0, b])\right)$ for all $\alpha \in\left(0, \frac{1}{2}\right)$ and $\beta \in\left(0, \frac{1-2 \alpha}{16}\right)$, as shown in the proof of property (i) of Theorem 3.4.

Property (ii) can be proved arguing exactly as in the proof of Theorem 3.5, replacing (3.24) by (3.31), with $\alpha=0$.

Finally, we establish (iii). To this aim, we note that if $\psi \in C^{3}\left(\mathbb{R}^{2} \backslash\{0\}\right)$ then $h(\cdot, t) \in H_{\#}^{5}(0, b)$ for almost every $t$. Indeed, this can be proved as in the final part of the proof of Theorem 3.8.

To simplify the notation we use $(\cdot)^{\prime}$ to denote differentiation with respect to $x$. Differentiating equation (3.31) with respect to $x$, estimating $Q_{x}$ by (4.1), one gets that for almost every $t \in\left(0, T_{0}\right)$

$$
\begin{aligned}
\int_{0}^{b}\left|h^{(\mathrm{v})}\right|^{2} d x & \leq C \int_{0}^{b}\left(1+h^{\prime \prime 8}+h^{\prime \prime 4} h^{\prime \prime \prime 2}+h^{\prime \prime \prime 4}+h^{\prime \prime 2}\left|h^{(\mathrm{iv})}\right|^{2}+\left|h^{(\mathrm{iv})}\right|^{2}+H^{\prime 2}\right) d x \\
& \leq C \int_{0}^{b}\left(1+h^{\prime \prime 8}+h^{\prime \prime \prime 4}+\left|h^{(\mathrm{iv})}\right|^{\frac{8}{3}}+H^{\prime 2}\right) d x
\end{aligned}
$$

Estimating

$$
\int_{0}^{b}\left(h^{\prime \prime 8}+h^{\prime \prime \prime 4}+\left|h^{(\mathrm{iv})}\right|^{\frac{8}{3}}\right) d x
$$

as in (4.15), and using Young's inequality to estimate the products of the $L^{2}$-norms of $h^{\prime \prime}$ and $h^{(\mathrm{v})}$, we arrive at

$$
\int_{0}^{b}\left|h^{(\mathrm{v})}\right|^{2} d x \leq \eta \int_{0}^{b}\left|h^{(\mathrm{v})}\right|^{2} d x+C_{\eta} \int_{0}^{b}\left(1+H^{\prime 2}\right) d x .
$$

By this inequality, taking $\eta$ sufficiently small and integrating in time, property (iii) follows.

We now come to main result of the section.

Theorem 4.3. Let $h_{0} \in H_{\#}^{3}(0, b), h_{0}>0$, let $\psi \in C^{4}\left(\mathbb{R}^{2} \backslash\{0\}\right)$, and let $T_{0}$ be as in Theorem 3.4. Then the solution to (3.1) constructed in Theorem 3.8 belongs to $H^{1}\left(0, T_{0} ; L^{2}(0, b)\right) \cap$ $L^{2}\left(0, T_{0} ; H^{6}(0, b)\right)$

Proof. We start by observing that (3.24) gives

$$
\begin{aligned}
\frac{h_{i, N}^{(\mathrm{iv})}}{J_{i, N}^{5}}= & 10 \frac{h_{i, N}^{\prime} h_{i, N}^{\prime \prime} h_{i, N}^{\prime \prime \prime}}{J_{i, N}^{7}}+\frac{5}{2} \frac{h_{i, N}^{\prime \prime 3}}{J_{i, N}^{7}}-\frac{35}{2} \frac{h_{i, N}^{\prime 2} h_{i, N}^{\prime \prime 3}}{J_{i, N}^{9}}+\psi_{x x}\left(-h_{i, N}^{\prime}, 1\right) \frac{h_{i, N}^{\prime \prime}}{\varepsilon} \\
& +\frac{1}{\varepsilon} H_{i, N}+\frac{2}{\varepsilon} c_{i, N}-\frac{1}{\varepsilon} Q_{i, N} \\
= & M_{i, N}+\frac{1}{\varepsilon} H_{i, N}+\frac{2}{\varepsilon} c_{i, N}-\frac{1}{\varepsilon} Q_{i, N},
\end{aligned}
$$

where we set

$$
M_{i, N}:=10 \frac{h_{i, N}^{\prime} h_{i, N}^{\prime \prime} h_{i, N}^{\prime \prime \prime}}{J_{i, N}^{7}}+\frac{5}{2} \frac{h_{i, N}^{\prime \prime 3}}{J_{i, N}^{7}}-\frac{35}{2} \frac{h_{i, N}^{\prime 2} h_{i, N}^{\prime \prime 3}}{J_{i, N}^{9}}+\psi_{x x}\left(-h_{i, N}^{\prime}, 1\right) \frac{h_{i, N}^{\prime \prime}}{\varepsilon} .
$$


Differentianting (4.26) with respect to $x$, recalling $(3.20)$, and setting $\frac{d}{d \sigma_{i, N}}:=\frac{1}{J_{i, N}} \frac{d}{d x}$, we get

$$
\left(\frac{h_{i, N}^{(\mathrm{iv})}}{J_{i, N}^{5}}\right)^{\prime}=M_{i, N}^{\prime}+\frac{J_{i-1, N}}{\varepsilon} \int_{0}^{x} \frac{h_{i, N}-h_{i-1, N}}{\Delta T} d \zeta-\frac{J_{i, N}}{\varepsilon} \mathbb{C} E\left(u_{i, N}\right): \frac{d}{d \sigma_{i, N}} E\left(u_{i, N}\right)
$$

Dividing both sides of the last identity by $J_{i-1, N}$ and differentiating again with respect to $x$, we get

$$
\left[\frac{1}{J_{i-1, N}}\left(\frac{h_{i, N}^{(\mathrm{iv})}}{J_{i, N}^{5}}\right)^{\prime}\right]^{\prime}=\left(\frac{M_{i, N}^{\prime}}{J_{i-1, N}}\right)^{\prime}+\frac{1}{\varepsilon} \frac{h_{i, N}-h_{i-1, N}}{\Delta T}-\left(\frac{J_{i, N}}{\varepsilon J_{i-1, N}} \mathbb{C} E\left(u_{i, N}\right): \frac{d}{d \sigma_{i, N}} E\left(u_{i, N}\right)\right)^{\prime}
$$

Expanding both sides of the above equation, we obtain

$$
\begin{aligned}
& \frac{h_{i, N}^{(\mathrm{vi})}}{J_{i, N}^{5} J_{i-1, N}}-\frac{10 h_{i, N}^{\prime} h_{i, N}^{\prime \prime} h_{i, N}^{(\mathrm{v})}}{J_{i, N}^{7} J_{i-1, N}}-\frac{5 h_{i, N}^{\prime \prime 2} h_{i, N}^{(\mathrm{iv})}}{J_{i, N}^{7} J_{i-1, N}}-\frac{5 h_{i, N}^{\prime} h_{i, N}^{\prime \prime \prime} h_{i, N}^{(\mathrm{iv})}}{J_{i, N}^{7} J_{i-1, N}}+\frac{35 h_{i, N}^{\prime 2} h_{i, N}^{\prime \prime 2} h_{i, N}^{(\mathrm{iv})}}{J_{i, N}^{9} J_{i-1, N}} \\
& -\frac{h_{i-1, N}^{\prime} h_{i-1, N}^{\prime \prime}}{J_{i-1, N}^{3}}\left(\frac{h_{i, N}^{(\mathrm{v})}}{J_{i, N}^{5}}-\frac{5 h_{i, N}^{\prime} h_{i, N}^{\prime \prime} h_{i, N}^{(\mathrm{iv})}}{J_{i, N}^{7}}\right)=\left(\frac{M_{i, N}^{\prime}}{J_{i-1, N}}\right)^{\prime}+\frac{1}{\varepsilon} \frac{h_{i, N}-h_{i-1, N}}{\Delta T} \\
& -\frac{1}{\varepsilon}\left[\frac{h_{i, N}^{\prime} h_{i, N}^{\prime \prime}}{J_{i, N} J_{i-1, N}}-\frac{h_{i-1, N}^{\prime} h_{i-1, N}^{\prime \prime} J_{i, N}}{J_{i-1, N}^{3}}\right]\left(\mathbb{C} E\left(u_{i, N}\right): \frac{d}{d \sigma_{i, N}} E\left(u_{i, N}\right)\right) \\
& -\frac{J_{i, N}}{\varepsilon J_{i-1, N}}\left[J_{i, N} \mathbb{C} E\left(u_{i, N}\right): \frac{d^{2}}{d \sigma_{i, N}^{2}} E\left(u_{i, N}\right)+J_{i, N} \mathbb{C} \frac{d}{d \sigma_{i, N}} E\left(u_{i, N}\right): \frac{d}{d \sigma_{i, N}} E\left(u_{i, N}\right)\right] .
\end{aligned}
$$

Let us now set

$$
\begin{aligned}
R_{i, N}:= & \frac{10 h_{i, N}^{\prime} h_{i, N}^{\prime \prime} h_{i, N}^{(\mathrm{v})}}{J_{i, N}^{7} J_{i-1, N}}+\frac{5 h_{i, N}^{\prime \prime 2} h_{i, N}^{(\mathrm{iv})}}{J_{i, N}^{7} J_{i-1, N}}+\frac{5 h_{i, N}^{\prime} h_{i, N}^{\prime \prime \prime} h_{i, N}^{(\mathrm{iv})}}{J_{i, N}^{7} J_{i-1, N}}-\frac{35 h_{i, N}^{\prime 2} h_{i, N}^{\prime \prime 2} h_{i, N}^{(\mathrm{iv})}}{J_{i, N}^{9} J_{i-1, N}} \\
& +\frac{h_{i-1, N}^{\prime} h_{i-1, N}^{\prime \prime}}{J_{i-1, N}^{3}}\left(\frac{h_{i, N}^{(\mathrm{v})}}{J_{i, N}^{5}}+\frac{5 h_{i, N}^{\prime} h_{i, N}^{\prime \prime} h_{i, N}^{(\mathrm{iv})}}{J_{i, N}^{7}}\right) .
\end{aligned}
$$

Multiplying both sides of $(4.28)$ by $h_{i, N}^{(\mathrm{vi})}$, integrating with respect to $x$, and recalling that all the $J_{i, N}$ are equibounded in $L^{\infty}$, after three integration by parts in the second integral, we have

$$
\begin{aligned}
C_{0} \int_{0}^{b}\left|h_{i, N}^{(\mathrm{vi})}\right|^{2} d x & +\frac{1}{\varepsilon} \int_{0}^{b} \frac{h_{i, N}^{\prime \prime \prime 2}-h_{i-1, N}^{\prime \prime \prime} h_{i, N}^{\prime \prime \prime}}{\Delta T} d x \leq C \int_{0}^{b}\left|h_{i, N}^{(\mathrm{vi})}\right|\left[\left|R_{i, N}\right|+\left|\left(\frac{M_{i, N}^{\prime}}{J_{i-1, N}}\right)^{\prime}\right|\right] d x \\
& +C \int_{0}^{b}\left(\left|h_{i, N}^{\prime \prime}\right|+\left|h_{i-1, N}^{\prime \prime}\right|\right)\left|h_{i, N}^{(\mathrm{vi})}\right|\left|D_{\sigma_{i, N}}\left(E\left(u_{i, N}\right)\right)\right| d x+C \int_{0}^{b}\left|h_{i, N}^{(\mathrm{vi})}\right|\left|D_{\sigma_{i, N}}\left(E\left(u_{i, N}\right)\right)\right|^{2} d x \\
& +C \int_{0}^{b}\left|h_{i, N}^{(\mathrm{vi})}\right|\left|D_{\sigma_{i, N} \sigma_{i, N}}^{2}\left(E\left(u_{i, N}\right)\right)\right| d x \\
\leq & \frac{C_{0}}{2} \int_{0}^{b}\left|h_{i, N}^{(\mathrm{vi})}\right|^{2} d x+C \int_{0}^{b}\left[\left|R_{i, N}\right|^{2}+\left|\left(\frac{M_{i, N}^{\prime}}{J_{i-1, N}}\right)^{\prime}\right|^{2}\right] d x+C \int_{0}^{b}\left(\left|h_{i, N}^{\prime \prime}\right|^{4}+\left|h_{i-1, N}^{\prime \prime}\right|^{4}\right) d x \\
& +C \int_{0}^{b}\left(\left|D_{\sigma_{i, N}}\left(E\left(u_{i, N}\right)\right)\right|^{4}+\left|D_{\sigma_{i, N} \sigma_{i, N}}^{2}\left(E\left(u_{i, N}\right)\right)\right|^{2}\right) d x
\end{aligned}
$$


Observing that $h_{i, N}^{\prime \prime \prime 2}-h_{i-1, N}^{\prime \prime \prime} h_{i, N}^{\prime \prime \prime} \geq \frac{1}{2}\left(h_{i, N}^{\prime \prime \prime 2}-h_{i-1, N}^{\prime \prime \prime 2}\right)$, recalling (4.2), and using Theorem 6.4 , we get

$$
\begin{aligned}
\frac{C_{0}}{2} \int_{0}^{b}\left|h_{i, N}^{(\mathrm{vi})}\right|^{2} d x & +\frac{1}{2 \varepsilon} \int_{0}^{b} \frac{\left(h_{i, N}^{\prime \prime \prime 2}-h_{i-1, N}^{\prime \prime \prime 2}\right)}{\Delta T} d x \leq C \int_{0}^{b}\left[\left|R_{i, N}\right|+\left|\left(\frac{M_{i, N}^{\prime}}{J_{i-1, N}}\right)^{\prime}\right|\right]^{2} d x \\
& +C \int_{0}^{b}\left(1+\left|h_{i, N}^{(\mathrm{v})}\right|^{2}+\left|h_{i-1, N}^{(\mathrm{v})}\right|^{2}\right) d x \\
\leq \eta \int_{0}^{b}\left(\left|h_{i, N}^{(\mathrm{vi})}\right|^{2}+\left|h_{i-1, N}^{(\mathrm{vi})}\right|^{2}\right) d x & +C_{\eta} \int_{0}^{b}\left(1+\left|h_{i, N}^{(\mathrm{v})}\right|^{2}+\left|h_{i-1, N}^{(\mathrm{v})}\right|^{2}\right) d x
\end{aligned}
$$

where in the last inequality we used Lemma 4.4 below. Summing up over $i$, we have

$$
\begin{aligned}
\frac{C_{0}}{2} \int_{0}^{T_{0}} \int_{0}^{b}\left|\frac{\partial^{6} h_{N}}{\partial x^{6}}\right|^{2} d x d t+ & \frac{1}{2 \varepsilon} \int_{0}^{b}\left(h_{N, N}^{\prime \prime \prime 2}-h_{0}^{\prime \prime \prime 2}\right) d x \\
& \leq 3 \eta \int_{0}^{T_{0}} \int_{0}^{b}\left|\frac{\partial^{6} h_{N}}{\partial x^{6}}\right|^{2} d x d t+C_{\eta} \int_{0}^{T_{0}} \int_{0}^{b}\left(1+\left|\frac{\partial^{4} h_{N}}{\partial x^{4}}\right|^{2}\right) d x .
\end{aligned}
$$

Choosing $\eta$ small enough and recalling (3.18), we conclude that

$$
\int_{0}^{T_{0}} \int_{0}^{b}\left|\frac{\partial^{6} h_{N}}{\partial x^{6}}\right|^{2} d x d t \leq C
$$

with $C>0$ independent of $N$. This shows that $h_{N}$ is bounded in $L^{2}\left(0, T_{0} ; H_{\#}^{6}(0, b)\right)$, therefore $h \in L^{2}\left(0, T_{0} ; H_{\#}^{6}(0, b)\right)$.

To conclude the proof, observe that from (4.28), and arguing as above, we have

$$
\begin{aligned}
& \int_{0}^{b}\left|\frac{h_{i, N}-h_{i-1, N}}{\Delta T}\right|^{2} d x \leq C \int_{0}^{b}\left|h_{i, N}^{(\mathrm{vi})}\right|^{2} d x+C \int_{0}^{b}\left[\left|R_{i, N}\right|^{2}+\left|\left(\frac{M_{i, N}^{\prime}}{J_{i-1, N}}\right)^{\prime}\right|^{2}\right] d x \\
& \quad+C \int_{0}^{b}\left(\left|h_{i, N}^{\prime \prime}\right|^{2}+\left|h_{i-1, N}^{\prime \prime}\right|^{2}\right)\left|D_{\sigma_{i, N}}\left(E\left(u_{i, N}\right)\right)\right|^{2} d x+C \int_{0}^{b}\left|D_{\sigma_{i, N}}\left(E\left(u_{i, N}\right)\right)\right|^{4} d x \\
& \quad+C \int_{0}^{b}\left|D_{\sigma_{i, N} \sigma_{i, N}}^{2}\left(E\left(u_{i, N}\right)\right)\right|^{2} d x
\end{aligned}
$$

Using (4.30), the right-hand side of this inequality and thus we conclude that

$$
\int_{0}^{T_{0}} \int_{0}^{b}\left|\frac{\partial h_{N}}{\partial t}\right|^{2} d x d t \leq C
$$

with $C>0$ independent of $N$. Hence, $h \in H^{1}\left(0, T_{0} ; L^{2}(0, b)\right)$.

Lemma 4.4. Let $M_{i, N}$ and $R_{i, N}$ be defined as in (4.27) and (4.29). Then for any $\eta>0$

$$
\int_{0}^{b}\left[\left|R_{i, N}\right|^{2}+\left|\left(\frac{M_{i, N}^{\prime}}{J_{i-1, N}}\right)^{\prime}\right|^{2}\right] d x \leq \eta \int_{0}^{b}\left(\left|h_{i, N}^{(\mathrm{vi})}\right|^{2}+\left|h_{i-1, N}^{(\mathrm{vi})}\right|^{2}\right) d x+C_{\eta} .
$$

Proof. Recalling Theorem 3.4-(i), we have

$$
\left|R_{i, N}\right|^{2} \leq C\left[\left|h_{i, N}^{(\mathrm{v})}\right|^{2}\left(h_{i, N}^{\prime \prime 2}+h_{i-1, N}^{\prime \prime 2}\right)+\left|h_{i, N}^{(\mathrm{iv})}\right|^{2}\left(h_{i, N}^{\prime \prime 4}+h_{i-1, N}^{\prime \prime 4}+h_{i, N}^{\prime \prime \prime 2}\right)\right] .
$$

Moreover, a lengthy but straightforward calculation together with property (i) of Theorem 3.4 leads to the estimate

$$
\begin{aligned}
\left|\left(\frac{M_{i, N}^{\prime}}{J_{i-1, N}}\right)^{\prime}\right|^{2} \leq & C\left[\left|h_{i, N}^{(\mathrm{v})}\right|^{2} h_{i, N}^{\prime \prime 2}+\left|h_{i, N}^{(\mathrm{iv})}\right|^{2}\left(h_{i, N}^{\prime \prime 4}+h_{i-1, N}^{\prime \prime 4}+h_{i, N}^{\prime \prime \prime 2}\right)\right. \\
& \left.+h_{i, N}^{\prime \prime \prime}\left(h_{i, N}^{\prime \prime 2}+h_{i-1, N}^{\prime \prime 2}\right)+h_{i, N}^{\prime \prime \prime 2}\left(h_{i, N}^{\prime \prime 6}+h_{i-1, N}^{\prime \prime 6}\right)+\left(h_{i, N}^{\prime \prime 10}+h_{i-1, N}^{\prime \prime 10}\right)\right] .
\end{aligned}
$$


Using (4.31) and the above estimate, we get by Young's inequality

$$
\int_{0}^{b}\left[\left|R_{i, N}\right|^{2}+\left|\left(\frac{M_{i, N}^{\prime}}{J_{i-1, N}}\right)^{\prime}\right|^{2}\right] d x \leq \int_{0}^{b}\left[\left|h_{i, N}^{(\mathrm{v})}\right|^{\frac{5}{2}}+\left|h_{i, N}^{(\mathrm{iv})}\right|^{\frac{10}{3}}+\left|h_{i, N}^{\prime \prime \prime}\right|^{5}+h_{i, N}^{\prime \prime 10}+h_{i-1, N}^{\prime \prime 10}\right] d x .
$$

By repeatedly using Theorem 6.4 and recalling property (ii) of Theorem 3.4, we get

$$
\begin{gathered}
\int_{0}^{b}\left[\left|h_{i, N}^{(\mathrm{v})}\right|^{\frac{5}{2}}+\left|h_{i, N}^{(\mathrm{iv})}\right|^{\frac{10}{3}}+\left|h_{i, N}^{\prime \prime \prime}\right|^{5}+h_{i, N}^{\prime \prime 10}+h_{i-1, N}^{\prime \prime 10}\right] d x \leq\left[\left\|h_{i, N}^{(\mathrm{vi})}\right\|_{L^{2}}^{\frac{31}{16}}\left\|h_{i, N}^{\prime \prime}\right\|_{L^{2}}^{\frac{9}{16}}+\left\|h_{i, N}^{(\mathrm{vi})}\right\|_{L^{2}}^{\frac{11}{6}}\left\|h_{i, N}^{\prime \prime}\right\|_{L^{2}}^{\frac{3}{2}}\right. \\
\left.+\left\|h_{i, N}^{(\mathrm{vi})}\right\|_{L^{2}}^{\frac{13}{8}}\left\|h_{i, N}^{\prime \prime}\right\|_{L^{2}}^{\frac{27}{8}}+\left\|h_{i, N}^{(\mathrm{vi})}\right\|_{L^{2}}\left\|h_{i, N}^{\prime \prime}\right\|_{L^{2}}^{9}+\left\|h_{i-1, N}^{(\mathrm{vi})}\right\|_{L^{2}}\left\|h_{i-1, N}^{\prime \prime}\right\|_{L^{2}}^{9}\right] \\
\leq \eta \int_{0}^{b}\left(\left|h_{i, N}^{(\mathrm{vi})}\right|^{2}+\left|h_{i-1, N}^{(\mathrm{vi})}\right|^{2}\right) d x+C_{\eta} .
\end{gathered}
$$

Combining the last estimate with (4.32) we obtain the thesis.

\section{UNIQUENESS}

In this section we show that if $\psi$ is of class $C^{3}$ away from the origin, then local weak solutions are unique. As before, $h_{0}$ satisfies (2.4).

Theorem 5.1 (Uniqueness). Let $\psi \in C^{3}\left(\mathbb{R}^{2} \backslash\{0\}\right)$, and let $h_{1}$, $h_{2}$ be two weak solutions of (3.1) in $\left[0, T_{0}\right]$ with initial datum $h_{0}$. Then $h_{1}=h_{2}$.

Proof. To simplify the notation, throughout the proof we use $(\cdot)^{\prime}$ to denote differentiation with respect to $x$. First we observe that by Theorem 4.2 and an approximation argument, it follows that if $h$ is a weak solution of (3.1), then the function $t \mapsto \int_{0}^{b} h^{\prime 2} d x$ has a weak derivative, which coincides for almost every $t$ with $-2 \int_{0}^{b}\left\langle\frac{\partial h}{\partial t}, h^{\prime \prime}\right\rangle d x$. Therefore, multiplying equations (3.3) for $h_{1}$ and $h_{2}$ by $h_{2}^{\prime \prime}-h_{1}^{\prime \prime}$ and integrating by parts, we get for almost every $t$

$$
\begin{aligned}
& -\frac{1}{2} \frac{\partial}{\partial t} \int_{0}^{b}\left|h_{2}^{\prime}-h_{1}^{\prime}\right|^{2} d x \\
& =\int_{0}^{b}\left[\varepsilon\left(\frac{h_{2}^{\prime \prime}}{J_{2}^{5}}\right)^{\prime \prime}+\frac{5 \varepsilon}{2}\left(\frac{h_{2}^{\prime \prime 2}}{J_{2}^{7}} h^{\prime}\right)^{\prime}+\left(\psi_{x}\left(-h_{2}^{\prime}, 1\right)\right)^{\prime}+Q_{2}\right]\left[\frac{h_{2}^{(\mathrm{iv})}-h_{1}^{(\mathrm{iv})}}{J_{2}}-\frac{\left(h_{2}^{\prime \prime \prime}-h_{1}^{\prime \prime \prime}\right) h_{2}^{\prime \prime} h_{2}^{\prime}}{J_{2}^{3}}\right] d x \\
& -\int_{0}^{b}\left[\varepsilon\left(\frac{h_{1}^{\prime \prime}}{J_{1}^{5}}\right)^{\prime \prime}+\frac{5 \varepsilon}{2}\left(\frac{h_{1}^{\prime \prime 2}}{J_{1}^{7}} h^{\prime}\right)^{\prime}+\left(\psi_{x}\left(-h_{1}^{\prime}, 1\right)\right)^{\prime}+Q_{1}\right]\left[\frac{h_{2}^{(\mathrm{iv})}-h_{1}^{(\mathrm{iv})}}{J_{1}}-\frac{\left(h_{2}^{\prime \prime \prime}-h_{1}^{\prime \prime \prime}\right) h_{1}^{\prime \prime} h_{1}^{\prime}}{J_{1}^{3}}\right] d x,
\end{aligned}
$$

where the subscripts $i$ refer to the functions $h_{i}, i=1,2$. After repeated use of Young's inequality, using the fact that $h_{i}^{\prime}$ is bounded, and that the function $t \mapsto\left(1+t^{2}\right)^{-\frac{m}{2}}, m>0$, is Lipschitz continuous, we get

$$
\begin{aligned}
& \int_{0}^{b}\left|h_{2}^{(\mathrm{iv})}-h_{1}^{(\mathrm{iv})}\right|^{2} d x+\frac{\partial}{\partial t} \int_{0}^{b}\left|h_{2}^{\prime}-h_{1}^{\prime}\right|^{2} d x \\
& \leq C \int_{0}^{b}\left[\left|h_{1}^{(\mathrm{iv})}\right|^{2}+\left|h_{2}^{(\mathrm{iv})}\right|^{2}+h_{1}^{\prime \prime 2} h_{1}^{\prime \prime \prime}+h_{2}^{\prime \prime 2} h_{2}^{\prime \prime \prime 2}+h_{1}^{\prime \prime 6}+h_{2}^{\prime \prime 6}+1\right]\left|h_{2}^{\prime}-h_{1}^{\prime}\right|^{2} d x \\
& \quad+C \int_{0}^{b}\left[h_{1}^{\prime \prime \prime 2}+h_{2}^{\prime \prime \prime 2}+h_{1}^{\prime \prime 4}+h_{2}^{\prime \prime 4}\right]\left|h_{2}^{\prime \prime}-h_{1}^{\prime \prime}\right|^{2} d x+C \int_{0}^{b}\left[h_{1}^{\prime \prime 2}+h_{2}^{\prime \prime 2}\right]\left|h_{2}^{\prime \prime \prime}-h_{1}^{\prime \prime \prime}\right|^{2} d x \\
& \quad+C \int_{0}^{b}\left|Q_{2}-Q_{1}\right|^{2} d x=: I_{1}+I_{2}+I_{3}+I_{4} .
\end{aligned}
$$


Setting $B(t):=\left\|h_{1}^{(\mathrm{v})}\right\|_{2}^{2}+\left\|h_{2}^{(\mathrm{v})}\right\|_{2}^{2}$, using Theorem 6.4 to estimate $\left\|h_{i}^{\prime \prime}\right\|_{\infty},\left\|h_{i}^{\prime \prime \prime}\right\|_{\infty},\left\|h_{i}^{(\mathrm{iv})}\right\|_{\infty}$ with $\left\|h_{i}^{(\mathrm{v})}\right\|_{2}$, and recalling that $\left\|h_{i}^{\prime \prime}\right\|_{2}$ is bounded, we have

$$
I_{1} \leq C(1+B(t)) \int_{0}^{b}\left|h_{2}^{\prime}-h_{1}^{\prime}\right|^{2} d x .
$$

Estimating $\left\|h_{i}^{\prime \prime}\right\|_{\infty},\left\|h_{i}^{\prime \prime \prime}\right\|_{\infty}$, with $\left\|h_{i}^{(\mathrm{v})}\right\|_{2}$ and using Theorem 6.4 again, we get by Young's inequality

$$
I_{2}+I_{3} \leq \eta \int_{0}^{b}\left|h_{2}^{(\mathrm{iv})}-h_{1}^{(\mathrm{iv})}\right|^{2} d x+C_{\eta}(1+B(t)) \int_{0}^{b}\left|h_{2}^{\prime}-h_{1}^{\prime}\right|^{2} d x .
$$

By Lemma 6.10 we get

$$
I_{4} \leq\left\|h_{2}-h_{1}\right\|_{C^{1, \alpha}([0, b])}^{2} \leq \int_{0}^{b}\left|h_{2}^{\prime \prime}-h_{1}^{\prime \prime}\right|^{2} d x \leq \eta \int_{0}^{b}\left|h_{2}^{(\mathrm{iv})}-h_{1}^{(\mathrm{iv})}\right|^{2} d x+C_{\eta} \int_{0}^{b}\left|h_{2}^{\prime}-h_{1}^{\prime}\right|^{2} d x .
$$

From this inequality, (5.1), (5.2), and (5.3), and choosing $\eta$ sufficiently small, we have

$$
\frac{\partial}{\partial t} \int_{0}^{b}\left|h_{2}^{\prime}-h_{1}^{\prime}\right|^{2} d x \leq C(1+B(t)) \int_{0}^{b}\left|h_{2}^{\prime}-h_{1}^{\prime}\right|^{2} d x
$$

Since $B \in L^{1}(0, T)$ by Theorem 4.2-(iii), using Gronwall's lemma we conclude that $\int_{0}^{b} \mid h_{2}^{\prime}-$ $\left.h_{1}^{\prime}\right|^{2} d x=0$ for every $t$. Hence, the conclusion follows.

Remark 5.2. From Theorem 5.1 we deduce that if $\psi \in C^{3}\left(\mathbb{R}^{2} \backslash\{0\}\right)$, then the solutions of the discrete time evolution problem constructed in Section 2 converge to the unique solution of (3.1). If, in addition, $\psi$ is of class $C^{4}$ away from the origin, then, in view of Theorem 4.3 we conclude that the unique weak solution of $(3.1)$ belongs to $H^{1}\left(0, T ; L^{2}(0, b)\right) \cap L^{2}\left(0, T ; H_{\#}^{6}(0, b)\right)$.

Remark 5.3. Combining Corollary 3.7 and Theorem 4.3, by interpolation we conclude that whole sequence $h_{N}$ constructed in (2.6) satisfies

$$
h_{N} \rightarrow h \quad \text { in } L^{2}\left(0, T ; H_{\#}^{5}(0, b)\right) .
$$

\section{Appendix}

Here we collect some interpolation inequalities and other auxiliary results that are used throughout the paper.

The next two theorems are particular cases of more general statements proved in [1]. Precisely, from [1, Theorem 5.2] we have

Theorem 6.1. Let $\Omega \subset \mathbb{R}^{n}$ be a bounded open set satisfying the cone condition. Let $1 \leq p \leq \infty$ and $j, m$ be two integers such that $0 \leq j \leq m$ and $m \geq 1$. Then there exists $C>0$ such that

$$
\left\|D^{j} f\right\|_{L^{p}(\Omega)} \leq C\left(\left\|D^{m} f\right\|_{L^{p}(\Omega)}^{\frac{j}{m}}\|f\|_{L^{p}(\Omega)}^{\frac{m-j}{m}}+\|f\|_{L^{p}(\Omega)}\right)
$$

for all $f \in W^{m, p}(\Omega)$. Moreover, if $n=1, \Omega$ is an interval, and $f \in W_{\#}^{m, p}(\Omega)$, then (6.1) holds in the stronger form

$$
\left\|D^{j} f\right\|_{L^{p}(\Omega)} \leq C\left\|D^{m} f\right\|_{L^{p}(\Omega)}^{\frac{j}{m}}\|f\|_{L^{p}(\Omega)}^{\frac{m-j}{m}} .
$$

Proof. Inequality (6.1) follows by combining inequalities (3) and (1) in [1, Theorem 5.2].

If $f$ is periodic in the interval $\Omega$, the proof of (6.2) is contained in [28].

Remark 6.2. If $p=1$ the conclusion of the above theorem holds also for $f \in W^{m-1,1}(\Omega)$ such that $D^{m-1} f \in B V(\Omega)$, provided that in (6.1) we replace $\left\|D^{m} f\right\|_{L^{1}(\Omega)}$ by the total variation $\left|D\left(D^{m-1} f\right)\right|(\Omega)$. This can be easily seen by a standard approximation argument.

The next interpolation result is essentially contained in [1, Theorem 5.8]. 
Theorem 6.3. Let $\Omega \subset \mathbb{R}^{n}$ be a bounded open set satisfying the cone condition. Let $1 \leq p \leq q<\infty$ if $m p \geq n$, and let $1 \leq p \leq q \leq \infty$ if $m p>n$. Then there exists $C>0$ such that

$$
\|f\|_{L^{q}(\Omega)} \leq C\left(\left\|D^{m} f\right\|_{L^{p}(\Omega)}^{\theta}\|f\|_{L^{p}(\Omega)}^{1-\theta}+\|f\|_{L^{p}(\Omega)}\right),
$$

for all $f \in W^{m, p}(\Omega)$, where $\theta:=\frac{n}{m p}-\frac{n}{m q}$. Moreover, if $n=1, \Omega$ is an interval, $f \in W_{\#}^{m, p}(\Omega)$, and either $f$ vanishes at the boundary or $\int_{\Omega} f d x=0,(6.3)$ holds in the stronger form

$$
\|f\|_{L^{q}(\Omega)} \leq C\left\|D^{m} f\right\|_{L^{p}(\Omega)}^{\theta}\|f\|_{L^{p}(\Omega)}^{1-\theta} .
$$

Proof. Inequality (6.3) follows by combining inequality (10) in [1, Theorem 5.8] with (1) in [1, Theorem 5.2].

If $f$ is periodic in the interval $\Omega$ and either $f$ vanishes at the boundary or $\int_{\Omega} f d x=0,(6.4)$ follows from inequality (10) [1, Theorem 5.8], observing that

$$
\|f\|_{W^{m, p}(\Omega)} \leq C\left\|D^{m} f\right\|_{L^{p}(\Omega)},
$$

as a straightforward application of the Poincaré inequality.

Combining Theorems 6.1 and 6.3 we have the following theorem.

Theorem 6.4. Let $\Omega \subset \mathbb{R}^{n}$ be a bounded open set satisfying the cone condition. Let $s, j$, and $m$ be integers such that $0 \leq s \leq j \leq m$. Let $1 \leq p \leq q<\infty$ if $(m-j) p \geq n$, and let $1 \leq p \leq q \leq \infty$ if $(m-j) p>n$. Then, there exists $C>0$ such that

$$
\left\|D^{j} f\right\|_{L^{q}(\Omega)} \leq C\left(\left\|D^{m} f\right\|_{L^{p}(\Omega)}^{\theta}\left\|D^{s} f\right\|_{L^{p}(\Omega)}^{1-\theta}+\left\|D^{s} f\right\|_{L^{p}(\Omega)}\right)
$$

for all $f \in W^{m, p}(\Omega)$, where

$$
\theta:=\frac{1}{m-s}\left(\frac{n}{p}-\frac{n}{q}+j-s\right)
$$

Moreover, if $n=1, \Omega$ is an interval, $f \in W_{\#}^{m, p}(\Omega)$, and either $f$ vanishes at the boundary or $\int_{\Omega} f d x=0,(6.5)$ holds in the stronger form

$$
\left\|D^{j} f\right\|_{L^{q}(\Omega)} \leq C\left\|D^{m} f\right\|_{L^{p}(\Omega)}^{\theta}\left\|D^{s} f\right\|_{L^{p}(\Omega)}^{1-\theta} .
$$

Proof. Since $(m-j) p \geq n$, we may apply Theorem 6.3 to $D^{j} f$ to obtain

$$
\left\|D^{j} f\right\|_{L^{q}(\Omega)} \leq C\left(\left\|D^{m} f\right\|_{L^{p}(\Omega)}^{\tilde{\theta}}\left\|D^{j} f\right\|_{L^{p}(\Omega)}^{1-\tilde{\theta}}+\left\|D^{j} f\right\|_{L^{p}(\Omega)}\right)
$$

where $\tilde{\theta}=\frac{n}{m-j}\left(\frac{1}{p}-\frac{1}{q}\right)$. On the other hand, by applying Theorem 6.1 to $D^{s} f$, we get

$$
\left\|D^{j} f\right\|_{L^{p}(\Omega)} \leq C\left(\left\|D^{m} f\right\|_{L^{p}(\Omega)}^{\frac{j-s}{m-s}}\left\|D^{s} f\right\|_{L^{p}(\Omega)}^{\frac{m-j}{m-s}}+\left\|D^{s} f\right\|_{L^{p}(\Omega)}\right) .
$$

Inserting this inequality in the right-hand side of (6.7) we get

$$
\left\|D^{j} f\right\|_{L^{q}(\Omega)} \leq C\left(\left\|D^{m} f\right\|_{L^{p}(\Omega}^{\theta}\left\|D^{s} f\right\|_{L^{p}(\Omega)}^{1-\theta}+\left\|D^{m} f\right\|_{L^{p}(\Omega)}^{\tilde{\theta}}\left\|D^{s} f\right\|_{L^{p}(\Omega)}^{1-\tilde{\theta}}+\left\|D^{j} f\right\|_{L^{p}(\Omega)} .\right.
$$

Since $\tilde{\theta} \leq \theta$, a simple application of the Young inequality shows that

$$
\left\|D^{m} f\right\|_{L^{p}(\Omega)}^{\tilde{\theta}}\left\|D^{s} f\right\|_{L^{p}(\Omega)}^{1-\tilde{\theta}} \leq C\left(\left\|D^{m} f\right\|_{L^{p}(\Omega}^{\theta}\left\|D^{s} f\right\|_{L^{p}(\Omega)}^{1-\theta}+\left\|D^{s} f\right\|_{L^{p}(\Omega)}\right) .
$$

Therefore, inserting this inequality in (6.9) and using (6.8) again, we get

$$
\left\|D^{j} f\right\|_{L^{q}(\Omega)} \leq C\left(\left\|D^{m} f\right\|_{L^{p}(\Omega}^{\theta}\left\|D^{s} f\right\|_{L^{p}(\Omega)}^{1-\theta}+\left\|D^{m} f\right\|_{L^{p}(\Omega)}^{\frac{j-s}{m-s}}\left\|D^{s} f\right\|_{L^{p}(\Omega)}^{\frac{m-j}{m-s}}+\left\|D^{s} f\right\|_{L^{p}(\Omega)}\right) .
$$

Oberving that $\frac{j-s}{m-s} \leq \theta$, another application of the Young inequality leads to

$$
\left\|D^{m} f\right\|_{L^{p}(\Omega)}^{\frac{j-s}{m-s}}\left\|D^{s} f\right\|_{L^{p}(\Omega)}^{\frac{m-j}{m-s}} \leq C\left(\left\|D^{m} f\right\|_{L^{p}(\Omega}^{\theta}\left\|D^{s} f\right\|_{L^{p}(\Omega)}^{1-\theta}+\left\|D^{s} f\right\|_{L^{p}(\Omega)}\right) .
$$


Hence, (6.5) follows. The proof of $(6.6)$ is obtained in a similar (but simpler) way by combining (6.2) and (6.4).

Remark 6.5. As in Remark 6.2, the conclusion of Theorem 6.4 holds for a function $f \in W^{m-1,1}(\Omega)$ such that $D^{m-1} f \in B V(\Omega)$, provided that in (6.5) we replace $\left\|D^{m} f\right\|_{L^{1}(\Omega)}$ by $\left|D\left(D^{m-1} f\right)\right|(\Omega)$.

Nest we prove some identities, which are used in the derivation of the Euler-Lagrange equation, for the energy functional (2.3).

Lemma 6.6. Let $g$ be the function introduced in (3.2). Then, for every $\theta \in(0,2 \pi) \backslash\{\pi\}$

$$
g(\theta)+g_{\theta \theta}(\theta)=\frac{\psi_{x x}(\cos \theta, \sin \theta)}{\sin ^{2} \theta} .
$$

Proof. Since $\psi$ is positively one-homogeneous, we have that $\psi(x, y)=x \psi_{x}(x, y)+y \psi_{y}(x, y)$. Therefore we have

$$
\begin{aligned}
g(\theta)+g_{\theta \theta}(\theta) & =\psi(\cos \theta, \sin \theta)+\psi_{x x}(\cos \theta, \sin \theta) \sin ^{2} \theta-2 \psi_{x y}(\cos \theta, \sin \theta) \sin \theta \cos \theta \\
& +\psi_{y y}(\cos \theta, \sin \theta) \cos ^{2} \theta-\psi_{x}(\cos \theta, \sin \theta) \cos \theta-\psi_{y}(\cos \theta, \sin \theta) \sin \theta \\
& =\psi_{x x}(\cos \theta, \sin \theta) \sin ^{2} \theta-2 \psi_{x y}(\cos \theta, \sin \theta) \sin \theta \cos \theta+\psi_{y y}(\cos \theta, \sin \theta) \cos ^{2} \theta .
\end{aligned}
$$

Since $\psi_{x}$ and $\psi_{y}$ are positively zero-homogeneous, we have

$$
x \psi_{x x}(x, y)+y \psi_{x y}(x, y)=0 \quad \text { and } \quad x \psi_{x y}(x, y)+y \psi_{y y}(x, y)=0 .
$$

Inserting these identities in the formula above, we get

$$
\left(g+g_{\theta \theta}\right)(\theta)=\psi_{x x}(\cos \theta, \sin \theta)+\psi_{y y}(\cos \theta, \sin \theta) .
$$

Again from (6.10) we have that $\psi_{x x}(\cos \theta, \sin \theta) \cos ^{2} \theta=\psi_{y y}(\cos \theta, \sin \theta) \sin ^{2} \theta$. Therefore,

$$
\psi_{x x}(\cos \theta, \sin \theta)+\psi_{y y}(\cos \theta, \sin \theta)=\frac{\psi_{x x}(\cos \theta, \sin \theta)}{\sin ^{2} \theta} .
$$

Lemma 6.7. The following identity holds

$$
k_{\sigma \sigma}+\frac{1}{2} k^{3}=-\left(\frac{h_{x x}}{J^{5}}\right)_{x x}-\frac{5}{2}\left(\frac{h_{x x}^{2}}{J^{7}} h_{x}\right)_{x}
$$

for $h$ sufficiently smooth, where $J:=\sqrt{1+h_{x}^{2}}$.

Proof. Since $\frac{\partial}{\partial x}=J \frac{\partial}{\partial \sigma}$,

$$
\begin{aligned}
\left(\frac{h_{x x}}{J^{5}}\right)_{x x}+\frac{5}{2}\left(\frac{h_{x x}^{2}}{J^{7}} h_{x}\right)_{x} & =-\left(\frac{k}{J^{2}}\right)_{x x}+\frac{5}{2}\left(\frac{k^{2}}{J} h_{x}\right)_{x} \\
& =\left(-\frac{k_{\sigma}}{J}-2 \frac{k^{2}}{J} h_{x}\right)_{x}+\frac{5}{2}\left(\left(k^{2}\right)_{x} \frac{h_{x}}{J}-k^{3}\right) \\
& =-k_{\sigma \sigma}-\frac{1}{2} k^{3} .
\end{aligned}
$$

The following lemma has been used in the proof of the existence theorem.

Lemma 6.8. Let $k \in \mathbb{N} \cup\{0\}$, let $h \in H_{\#}^{2}(0, b)$, and let $f \in L^{2}(0, b)$ be such that $\int_{0}^{b} f \varphi^{(k)} d x=0$ for all $\varphi \in C_{c}^{k}(0, b)$ with $\int_{0}^{b} \varphi d x=0$ and $\int_{\Gamma_{h}} \int_{0}^{x} \varphi d \zeta d \mathcal{H}^{1}=0$. Then if $k \geq 1$

$$
f(x)=a \int_{0}^{x} \int_{0}^{x_{k}} \cdots \int_{0}^{x_{1}} \sqrt{1+\left(h^{\prime}(r)\right)^{2}} d r \ldots d x_{k-1} d x_{k}+P_{k}(x)
$$


for some $a \in \mathbb{R}$ and some polynomial $P_{k}$ of degree $k$, and if $k=0$

$$
f(x)=a \int_{0}^{x} \sqrt{1+\left(h^{\prime}(r)\right)^{2}} d r+a_{0}
$$

for some $a_{0}, a \in \mathbb{R}$.

Proof. Let $\chi \in C_{c}^{k+2}(0, b)$ and set $\varphi_{\varepsilon}(x):=\left(\frac{\chi^{\prime}(x)}{J_{\varepsilon}(x)}\right)^{\prime}$, where $J_{\varepsilon}(x):=\sqrt{1+h_{\varepsilon}^{\prime 2}(x)}$ and $h_{\varepsilon}$ is the standard mollification of $h$.

Let $\psi \in C_{c}^{\infty}(0, b)$ be such that

$$
\int_{0}^{b} \psi d x=0, \quad \int_{\Gamma_{h}} \int_{0}^{x} \psi d \zeta d \mathcal{H}^{1}=1
$$

and define

$$
\psi_{\varepsilon}:=\varphi_{\varepsilon}-c_{\varepsilon} \psi
$$

where $c_{\varepsilon}:=\int_{\Gamma_{h}} \int_{0}^{x} \varphi_{\varepsilon} d \zeta d \mathcal{H}^{1}$. Note that by construction $\psi_{\varepsilon} \in C_{c}^{k}(0, b)$ and satisfies $\int_{0}^{b} \psi_{\varepsilon} d x=0$ and $\int_{\Gamma_{h}} \int_{0}^{x} \psi_{\varepsilon} d \zeta d \mathcal{H}^{1}=0$. Hence,

$$
\int_{0}^{b}\left(\frac{\chi^{\prime}}{J_{\varepsilon}}\right)^{(k+1)} f d x=c_{\varepsilon} \int_{0}^{b} f \psi^{(k)} d x .
$$

If $f$ is smooth then the previous identity is equivalent to

$$
(-1)^{k+2} \int_{0}^{b}\left(\frac{f^{(k+1)}}{J_{\varepsilon}}\right)^{\prime} \chi d x=c_{\varepsilon} \int_{0}^{b} f \psi^{(k)} d x
$$

and letting $\varepsilon \rightarrow 0$, we obtain

$$
\int_{0}^{b}\left(\frac{f^{(k+1)}}{J}\right)^{\prime} \chi d x=0
$$

for all $\chi \in C_{c}^{k+2}(0, b)$ and the conclusion follows. The general case is obtained via a standard approximation argument.

The following trace theorem is a particular case of [27, Theorem 15.17].

Theorem 6.9. Let $\Omega \subset \mathbb{R}_{+}^{2}$ be a bounded Lipschitz open set and $S=\partial \Omega \cap\{y=0\}$. If $1<p<2$, then for all $u \in W^{1, p}(\Omega)$, denoting by $T r$ the trace operator, we have

$$
\|\operatorname{Tr}(u)\|_{L^{\frac{p}{2-p}(S)}} \leq C\|u\|_{W^{1, p}(\Omega)}
$$

for a suitable constant $C>0$.

We conclude the appendix with the following elliptic estimate.

Lemma 6.10. Let $M>0, c_{0}>0$. Let $h_{1}, h_{2} \in A P \cap C_{\#}^{1, \alpha}([0, b])$ for some $\alpha \in(0,1)$, with $\left\|h_{i}\right\|_{C^{1, \alpha}([0, b])} \leq M$ and $h_{i} \geq c_{0}$, and let $u_{1}$ and $u_{2}$ the corresponding elastic equilibria in $\Omega_{h_{1}}$ and $\Omega_{h_{2}}$, respectively. Then,

$$
\| E\left(u_{1}\left(\cdot, h_{1}(\cdot)\right)-E\left(u_{2}\left(\cdot, h_{2}(\cdot)\right)\left\|_{C^{1, \alpha}([0, b])} \leq C\right\| h_{1}-h_{2} \|_{C^{1, \alpha}([0, b])}\right.\right.
$$

for some constant $C>0$ depending only on $M, c_{0}$, and $\alpha$.

Proof. Recall that by minimality

$$
\int_{\Omega_{h_{1}}} \mathbb{C} E\left(u_{1}\right): E(w) d z=0, \quad \int_{\Omega_{h_{2}}} \mathbb{C} E\left(u_{2}\right): E(w) d z=0
$$


for all $w \in L D_{\#}\left(\Omega_{h_{i}} ; \mathbb{R}^{2}\right)$ with $w(x, 0)=0$. Let $\Phi: \Omega_{h_{1}} \rightarrow \Omega_{h_{2}}$ be a diffeomorphism such that $\|\Phi-I d\|_{C^{1, \alpha}\left(\Omega_{h_{1}}\right)} \leq C\left\|h_{2}-h_{1}\right\|_{C^{1, \alpha}([0, b])}$. Then, it is easily checked that

$$
\int_{\Omega_{h_{1}}} \mathbb{C} E\left(u_{2} \circ \Phi^{-1}\right): E(w) d z=\int_{\Omega_{h_{1}}} d: \nabla w d z
$$

with $\|d\|_{C^{0, \alpha}\left(\Omega_{h_{1}}\right)} \leq C\left\|h_{2}-h_{1}\right\|_{C^{1, \alpha}([0, b])}$. Setting $v:=u_{1}-u_{2} \circ \Phi^{-1}$ it follows that

$$
\int_{\Omega_{h_{1}}} \mathbb{C} E(v): E(w) d z=-\int_{\Omega_{h_{1}}} d: \nabla w d z .
$$

From standard elliptic regularity (see [21, Proposition 8.9]), it follows that

$$
\|v\|_{C^{1, \alpha}\left(\Omega_{h_{1}}\right)} \leq C\left(\|v\|_{H^{1}\left(\Omega_{h_{1}}\right)}+\|d\|_{C^{0, \alpha}\left(\bar{\Omega}_{h_{1}}\right)}\right),
$$

for some constant $C>0$ depending only $M, c_{0}$, and $\alpha$. Since by (6.12), using also Korn's and Poincaré inequality, we have $\|v\|_{H^{1}\left(\Omega_{h_{1}}\right)} \leq C\|d\|_{C^{0, \alpha}\left(\bar{\Omega}_{h_{1}}\right)}$, the conclusion follows.

\section{ACKNOWLEDGMENT}

The authors warmly thank the Center for Nonlinear Analysis (NSF Grants No. DMS-0405343 and DMS-0635983), where part of this research was carried out. The research of I. Fonseca was partially funded by the National Science Foundation under Grants No. DMS-0401763 and DMS0905778 and that of G. Leoni under Grants No. DMS-0708039 and DMS-1007989. I. Fonseca and G. Leoni also acknowledge support of the National Science Foundation under the PIRE Grant No. OISE-0967140. The research of N. Fusco was also supported by the 2008 ERC under FP7, Advanced Grant N. 226234 in "Analytic Techniques for Geometric and Functional Inequalities". The authors would like to thank P. Piovano for carefully reading the manuscript.

\section{REFERENCES}

[1] Adams R.A.; Fournier J.F., Sobolev Spaces (second edition) Pure and Applied Mathematics (Amsterdam), 140. Elsevier/Academic Press, Amsterdam, 2003.

[2] Agmon S., Lectures on elliptic boundary value problems. Van Nostrand Mathematical Studies 2, D. Van Nostrand Co. Inc., Princeton, N.J., 1965.

[3] Almgren F.; Taylor J. E.; Wang L., Curvature-driven flows: a variational approach. SIAM J. Control Optim. 31 (1993), 387-438.

[4] Ambrosio L., Minimizing movements. Rend. Accad. Naz. Sci. XL Mem. Mat. Appl. 5 (1995), 191-246.

[5] Ambrosio L.; Fusco N.; Pallara D., Functions of bounded variation and free discontinuity problems. Oxford Mathematical Monographs. The Clarendon Press, Oxford University Press, New York, 2000.

[6] Ambrosio L.; Gigli, N.; Savaré, G. Gradient flows in metric spaces and in the space of probability measures. Second edition. Lectures in Mathematics ETH Zrich. Birkhuser Verlag, Basel, 2008.

[7] Angenent S.; Gurtin M.E., Multiphase thermomechanics with interfacial structure. II. Evolution of an isothermal interface. Arch. Rational Mech. Anal. 108 (1989), 323-391.

[8] Bellettini G.; Caselles V.; Chambolle A.; Novaga M., Crystalline mean curvature flow of convex sets. Arch. Ration. Mech. Anal. 179 (2006), 109-152.

[9] Bellettini G.; Mantegazza C.; Novaga M., Singular perturbations of mean curvature flow. J. Differential Geom. 75 (2007), 403-431.

[10] Bonnetier E.; Chambolle A., Computing the equilibrium configuration of epitaxially strained crystalline films. SIAM J. Appl. Math. 62 (2002), 1093-1121.

[11] Burger M.; Hausser H.; Stöcker C.; Voigt A., A level set approach to anisotropic flows with curvature regularization. J. Comput. Phys. 225 (2007), 183-205.

[12] Cahn J. W.; TAYlor J. E., Overview N0-113 - Surface motion by surface-diffusion. Acta Metallurgica et Materialia, 42 (1994), 1045-1063.

[13] Caselles V.; Chambolle A., Anisotropic curvature-drive flow of convex sets. Nonlinear Anal. 65 (2006), $1547-1577$ 
[14] Chen X., The Hele-Shaw problem and area-preserving curve-shortening motions. Arch. Rational Mech. Anal. 123 (1993), 117-151.

[15] Di Carlo A.; Gurtin M. E.; Podio-Guidugli P., A regularized equation for anisotropic motion-by-curvature. SIAM J. Appl. Math. 52 (1992), 1111-1119.

[16] Elliott C. M.; Garcke H., Existence results for diffusive surface motion laws. Adv. Math. Sci. Appl. 7 (1997), 467-490.

[17] Escher J.; Mayer U. F.; Simonett G., The surface diffusion flow for immersed hypersurfaces. SIAM J. Math. Anal. 29 (1998), 1419-1433.

[18] Fonseca I.; Fusco N.; Leoni G.; Millot V., Material voids for anisotropic surface energies. Work in progress.

[19] Fonseca I.; Fusco N.; Leoni G.; Morini M., Equilibrium configurations of epitaxially strained crystalline films: existence and regularity results. Arch. Rational Mech. Anal. 186 (2007), 477-537.

[20] Fung Y.C., A first course in Continuum Mechanics. Prentice-Hall, Inc, Englewood Cliffs, N.J., 1969.

[21] Fusco N.; Morini M., Equilibrium configurations of epitaxially strained elastic films: second order minimality conditions and qualitative properties of solutions. Preprint 2009.

[22] Garcke H., On Cahn-Hilliard systems with elasticity. Proc. Roy. Soc. Edinburgh Sect. A 133 (2003), $307-331$.

[23] Garcke H., On a Cahn-Hilliard model for phase separation with elastic misfit. Ann. Inst. H. Poincaré Anal. Non Linéaire 22 (2005), 165-185.

[24] Gurtin M. E.; JabBour M. E., Interface evolution in three dimensions with curvature-dependent energy and surface diffusion: interface-controlled evolution, phase transitions, epitaxial growth of elastic films. Arch. Ration. Mech. Anal. 163 (2002), 171-208.

[25] Gurtin M. E.; Soner H. M.; Souganidis P. E., Anisotropic motion of an interface relaxed by the formation of infinitesimal wrinkles. J. Differential Equations 119 (1995), 54-108.

[26] Herring C., Some theorems on the free energies of crystal surfaces., Physical Review 82 (1951), 87-93.

[27] Leoni G., A First Course in Sobolev Spaces. Graduate Studies in Mathematics. Vol 105. Am. Math. Soc. Providence, Rhode Island, 2009.

[28] LEONi G., Interpolation for intermediate derivatives http://www.ams.org/publications/authors/books/postpub /gsm-105

[29] Mantegazza C., Smooth geometric evolutions of hypersurfaces. Geom. Funct. Anal. 12 (2002), $138-182$.

[30] Mullins W. W., Solid surface morphologies governed by capillarity. In: Metal Surfaces. American society for metals, 1963.

[31] Rätz A.; Ribalta A.; Voigt A., Surface evolution of elastically stressed films under deposition by a diffuse interface model. J. Comp. Phys. 214 (2006), 187-208.

[32] Siegel M.; Miksis M. J.; Voorhees P. W., Evolution of material voids for highly anisotropic surface energy. J. Mech. Phys. Solids 52 (2004), 1319-1353.

(I. Fonseca) Department of Mathematical Sciences, Carnegie Mellon University, Pittsburgh, PA, U.S.A.

E-mail address, I. Fonseca: fonseca@andrew.cmu.edu

(N. Fusco) Dipartimento di Matematica e Applicazioni "R. Caccioppoli", Università degli Studi di NAPoli "Federico II" , NAPOli, ItAly

E-mail address, N. Fusco: n.fusco@unina.it

(G. Leoni) Department of Mathematical Sciences, Carnegie Mellon University, Pittsburgh, PA, U.S.A.

E-mail address, G. Leoni: giovanni@andrew.cmu.edu

(M. Morini) Dipartimento di Matematica, Università degli Studi di Parma , Parma, Italy

E-mail address, M. Morini: massimiliano.morini@unipr.it 